\title{
Extracción de recursos naturales en contextos de abundancia y escasez: Un análisis experimental sobre infracciones a cuotas en áreas de manejo y explotación de recursos bentónicos en el centro-sur de Chile* \\ Extraction of natural resources in contexts of abundance and scarcity: An experimental analysis on non-compliance with quotas in management and exploitation areas of benthic resources in central-southern Chile
}

\author{
OSCAR SANTIS** \\ CARlos CHÁvEZ***
}

\begin{abstract}
Resumen
Estudiamos, por medio de un experimento económico de campo contextualizado, los efectos de cambios exógenos en los niveles de abundancia de un recurso natural renovable en las decisiones de cumplimiento individual de usuarios operando bajo un régimen de propiedad común y un sistema de derechos de uso territorial pesqueros (DUTP), que considera cuotas de extracción y fiscalización externa para detectar y sancionar transgresiones. Nuestros resultados indican que, en presencia de abundancia del recurso, los pescadores disminuyen el nivel de extracción cuando enfrentan una cuota con fiscalización externa vis-à-vis un régimen de propiedad común, mientras que en situación de escasez del recurso se observa también una disminución en los niveles de extracción; sin embargo, en este caso los pescadores presentan niveles de incumplimiento y extracciones más altas en comparación con el escenario de abundancia, incluso con extracciones sobre el equilibrio de Nash.
\end{abstract}

Clasificación JEL: Q48, H23, Q53, H31.

Palabras clave: Experimentos económicos, cumplimiento, abundancia, derechos de uso territorial.

* Los autores agradecen el apoyo financiero y técnico para esta investigación recibido del Programa de Economía Ambiental para América Latina y el Caribe (LACEEP). Chávez reconoce y agradece el financiamiento parcial recibido a través del proyecto NS 100007, de la Iniciativa Científica Milenio, del Ministerio de Economía, Fomento y Turismo del Gobierno de Chile y a través de CONICYT/FONDAP/15110027. Agradecemos también a dos evaluadores anónimos de Estudios de Economía por los detallados y útiles comentarios y sugerencias.

** Programa Magíster en Economía de Recursos Naturales y del Medio Ambiente, Universidad de Concepción. E-mail: osantis@udec.cl

*** Departamento de Economía, Universidad de Concepción, Núcleo de Investigación en Economía Ambiental y de Recursos Naturales y Centro Interdisciplinario para la Investigación en Acuicultura (INCAR). E-mail: cchavez@udec.cl 


\begin{abstract}
We study through framed field economic experiment the effects of exogenous changes in abundance levels of a renewable natural resource on compliance individual decisions of users operating under a common property regime and a system of Territorial use rights in fisheries (TURF) considering extraction quotas and external enforcement to detect and sanctions violations. Our results indicate that in the presence of resource abundance, fishermen decrease the level of abstraction when they face a quota external control vis-à-vis a common property regime, while in a situation of resources scarcity observed also a reduction in extraction levels; however, in this case fishermen have levels of non compliance and extractions higher than in the scenario of resource abundance, even with extractions above the Nash equilibrium.
\end{abstract}

JEL Classification: $Q 48, H 23, Q 53, H 31$.

Keywords: Economics experiments, compliance, abundance, territorial user rights.

\title{
1. INTRODUCCIÓN
}

El uso de sistemas de manejo descentralizados de la actividad pesquera basados en derechos de propiedad está ganando creciente atención entre los administradores pesqueros. En el contexto de pesquerías artesanales, uno de estos sistemas incluye la creación y asignación de Derechos de Uso Territorial Pesqueros (DUTP). En este sistema se asignan derechos al uso o explotación de parte o la totalidad de recursos naturales localizados en un espacio geográfico definido. Los DUTP pueden ser designados sobre la columna de agua superficial, columna de agua intermedia, fondo marino, o una combinación de los mismos. En este sistema los derechos pueden ser asignados a agentes individuales, grupos de individuos organizados, o comunidades costeras (Wilen et al., 2012).

Una aplicación relevante de implementación de un sistema de DUTP en Chile lo constituyen las Áreas de Manejo y Explotación de Recursos Bentónicos (AMERB). Bajo este sistema se asignan derechos exclusivos de uso y explotación de recursos bentónicos de sectores geográficos ubicados en la franja costera de las cinco millas reservadas a la pesca artesanal o en aguas terrestres interiores, a organizaciones de pescadores artesanales legalmente constituidas (Castilla, 2010; Gelcich, 2010; Gelcich, 2006; González, 1996). ${ }^{1}$

1 Según datos de la Subsecretaría de Pesca (SUBPESCA), a marzo de 2013 el número de áreas de manejo decretadas a nivel nacional alcanzaba a 773 de las cuales 512 están asignadas actualmente, existiendo además 354 AMERB en trámite. En la Región del Biobío, a la misma fecha se decretaron 76 áreas de manejo, de las cuales 56 están actualmente asignadas, además de 24 AMERB en tramitación. En cuanto a la superficie decretada, la Región del Biobío cuenta con 26.397,17 hectáreas, correspondientes a un 23\% del total nacional. 
La literatura existente sugiere que, en general, el sistema AMERB ha tenido impacto económico positivo en algunas de las organizaciones signatarias (Castilla et al., 2007; Gelcich et al., 2010; González et al., 2006; San Martín et al., 2010; entre otros). Sin embargo, el referido sistema no ha estado exento de problemas. Entre otras dificultades destaca, primero, la presencia de extracción ilegal de recursos, un elemento clave que podría estar afectando seriamente el funcionamiento de esta medida de administración (Aburto et al., 2013; Chávez et al., 2010; Stotz et al., 2006; Stotz et al., 2008; San Martín et al., 2010, entre otros). Un segundo problema, que podría estar relacionado con el anterior, es la heterogeneidad en términos de productividad biológica de las áreas asignadas, esto es, mientras existen áreas con altos niveles de abundancia de recursos objetivos, otras mantienen niveles bajos de stocks que no se han recuperado (Stotz et al., 2006; Stotz et al., 2008).

La literatura existente no es concluyente respecto de cómo las condiciones de abundancia y escasez de un recurso natural renovable modifican el comportamiento de los usuarios que operan bajo un régimen de propiedad común y un sistema de derechos de uso territorial pesqueros (DUTP). Este trabajo presenta los resultados de un experimento económico de campo contextualizado cuyo objetivo es explorar los efectos de las diferencias en productividad biológica entre AMERB sobre el comportamiento infractor cuando, en el contexto de DUTP, existe una regulación de cuotas, y la fiscalización externa es insuficiente para inducir perfecto cumplimiento.

Los sujetos experimentales son pescadores artesanales de la Región del Biobío, en la zona centro-sur de Chile, miembros de organizaciones participantes en el sistema de AMERB que extraen principalmente el recurso loco ${ }^{2}$ (Concholepas concholepas). Las AMERB administradas por estos pescadores presentan distintos niveles de abundancia de este recurso. En este sentido, los sujetos participantes en el experimento están familiarizados con este tipo de contextos (abundancia y escasez). Siguiendo la taxonomía presentada por Harrison y List (2004), nuestro análisis está basado en el diseño y aplicación de un experimento de campo contextualizado (framed field experiment), con dos tratamientos. En el primer tratamiento se presenta un escenario con alta productividad biológica (abundancia) de la especie objetivo loco. En el segundo tratamiento se presenta un escenario con bajo nivel de abundancia o productividad biológica (escasez) de la especie objetivo loco. En ambos casos los sujetos experimentales enfrentan dos etapas de decisión. En la primera etapa, conocida como Línea Base Recurso de Propiedad Común (RPC), no existe ninguna regulación externa o interna asociada a la organización, y consecuentemente, los individuos seleccionan libremente el nivel de extracción. En la segunda etapa, denominada Fiscalización Formal, se impone un tratamiento que introduce cuotas de extracción, actividad de vigilancia de parte de una autoridad central, y la imposición de sanciones en caso que una infracción de cuota es detectada.

Se espera que los pescadores respondan a cambios en la abundancia de recursos en términos de extracción y comportamiento infractor. Cambios en

2 Gasterópodo de gran importancia para las comunidades costeras por su alto valor comercial, que por su sobreexplotación impulsó la instauración del sistema de AMERB a finales de los años 90 . 
los niveles de abundancia de recursos naturales debieran generar cambios en el comportamiento de sujetos regulados porque ellos podrían modificar los márgenes relevantes en la decisión de cumplimiento. Por ejemplo, incrementos en los niveles de abundancia podrían reducir los costos de extracción, si tales costos son dependientes del stock, en dichos casos se esperaría un incremento en niveles de extracción individual y agregados tanto en circunstancias de ausencia de regulación (recursos de propiedad común), como también en el caso en que existan controles externos.

Evidencia sobre las consecuencias de cambios en la abundancia de recursos en las decisiones de extracción individual ha sido reportada basados en la aplicación de diferentes diseños experimentales en juegos de RPC (Blanco et al., 2011; Maldonado y Moreno-Sánchez, 2009; Osés-Eraso y Viladrich-Grau, 2007; Osés-Eraso et al., 2008). Algunos experimentos se han centrado en explorar las respuestas de los usuarios a cambios exógenos en la disponibilidad del recurso, por ejemplo Osés-Eraso y Viladrich-Grau (2007) introducen un nivel definido de stock (de acuerdo con el tratamiento de abundancia, escasez o línea base) que mantienen durante todo el experimento, observando que cuando el recurso común es escaso se reduce el nivel de extracción. En un diseño similar, Blanco et al. (2011) introducen variaciones en los niveles de stock a lo largo del experimento, sin embargo, a diferencia de la investigación descrita con anterioridad, sus resultados sugieren que los usuarios enfrentados a etapas en donde el stock disminuye en forma moderada toman un tiempo (un par de rondas) para reducir sus extracciones, pero aumentan sus extracciones inmediatamente cuando el recurso se reduce a un tamaño en el que se pueden agotar por completo. Otros experimentos han explorado la combinación de cambios exógenos introducidos por el experimentalista y cambios endógenos de acuerdo con las decisiones de extracción de los usuarios. ${ }^{3}$

En nuestro diseño hemos optado por un experimento estático en beneficio de la comprensión de los sujetos experimentales, especialmente si consideramos las posibles dificultades que puede presentar un diseño dinámico, de mayor complejidad, aplicado en comunidades pesqueras en los cuales se presentan bajos niveles de escolaridad. En nuestro caso, tanto el escenario de abundancia como escasez presenta una solución interior para el equilibrio de Nash a diferencia de Maldonado y Moreno-Sánchez (2009) que presentan una solución de esquina en el caso de abundancia y otra interior en el caso de escasez.

En nuestros resultados el comportamiento de los pescadores enfrentados a escenarios de abundancia y escasez presenta diferencias significativas. En situaciones de abundancia cuando se introduce control de cuota y fiscalización exógena, los pescadores disminuyen sus extracciones respecto de la línea base RPC, presentando menores niveles de extracción e incumplimiento, mientras que

\footnotetext{
Por ejemplo, Maldonado y Moreno-Sánchez (2009) muestran que bajo condiciones de escasez los usuarios sobreextraen el recurso (pescadores), tomando decisiones por encima del equilibrio de Nash, sin embargo con la introducción de reglas de manejo la sobreexplotación se corrige. Por su parte Osés-Eraso et al. (2008) observan que si bien los usuarios expuestos a recursos iniciales más escasos (escasez exógena) aumentan sus extracciones, mientras más grande es la escasez impulsada por la acción de los propios usuarios de los recursos (escasez endógena) mayor será el nivel de extracción.
} 
en situación de escasez si bien los individuos también disminuyen sus extracciones cuando enfrentan una cuota con fiscalización externa, los pescadores presentan niveles de incumplimiento y extracciones más altas si son comparados con el escenario de abundancia, incluso con extracciones sobre el equilibrio de Nash.

El trabajo se organiza de la siguiente manera. En la sección 2 presentamos una descripción del sistema de derechos de uso territorial en pesquerías chilenas. La sección 3 presenta las principales hipótesis que deseamos evaluar a través de nuestros experimentos económicos de campo. La sección 4 incluye una descripción del diseño experimental y procedimientos. La sección 5 presenta y discute los resultados de la aplicación de los experimentos de campo a pescadores miembros de organizaciones participantes en el sistema de AMERB en el centro-sur de Chile. Finalmente, en la sección 6 presentamos las principales conclusiones de nuestro trabajo.

\section{EL SISTEMA DE DERECHOS DE USO TERRITORIAL EN PESQUERÍAS CHILENAS}

El sistema de derechos de uso territorial en pesquerías artesanales chilenas fue introducido legalmente en la Ley General de Pesca y Acuicultura de 1991; sin embargo, su aplicación solo comenzó hasta 1997, luego de la promulgación de su reglamento en $1995 .{ }^{4}$ Las AMERB pueden ser solicitadas por las comunidades de pescadores artesanales que establecen una organización legalmente constituida (sindicatos, asociaciones gremiales, cooperativas o comunidades indígenas) y presentan una propuesta de proyecto de manejo y explotación, que debe incluir, en primer lugar, un estudio de situación base (ESBA) que contenga la individualización del área (con indicación de su superficie y las respectivas coordenadas, de acuerdo con el decreto que la establece), que describa los recursos bentónicos existentes en el área (en términos de especies, cantidades, tipos de sustrato presentes en el fondo y su profundidad, etc.) y una descripción socioeconómica de la organización solicitante y de la localidad en que se inserta, y en segundo lugar, un plan de manejo y explotación (PME) especificando, entre otras cosas, un conjunto de acciones dirigidas a garantizar el manejo sostenible de la pesquería. Este PME incluye un plan de explotación anual del área solicitada, especificando la modalidad y periodos de explotación, así como los criterios aplicados para determinar las cantidades que se extraerán de las especies principales (Villena y Chávez, 2005). ${ }^{5}$

4 Los objetivos principales asociados con la asignación de las AMERB son: (i) propender a la conservación de los recursos bentónicos y sustentabilidad de la actividad artesanal; (ii) mantener o incrementar la productividad biológica de los recursos bentónicos; e (iii) incentivar y promover el manejo participativo (SUBPESCA, 2000. Esto sugiere que con este sistema se deseaba reducir el esfuerzo pesquero global y mejorar la situación económica de los pescadores artesanales.

5 Según el Reglamento sobre Áreas de Manejo y explotación de Recursos Bentónicos, DS No 355 de 1995 (última modificación, D.S. No 49 de 2009), la elaboración del proyecto y la ejecución del plan de manejo y explotación de recursos bentónicos deberán contar con la asistencia de una institución ejecutora, que deberá tener la forma orgánica de Universidades o Institutos de Investigación, estatales o privados, o de empresas consultoras 
La explotación de las AMERB se controla y opera basándose en el total de la cuota global anual autorizada llamada Captura Total Permisible (CTP), que consiste en una fracción del número total de individuos maduros disponibles de una especie en el área. Por consiguiente, cada año las organizaciones asignatarias de las AMERB deben contratar los servicios de universidades o consultores registrados para realizar un estudio de seguimiento, en el cual se definen las capturas totales permisibles para cada especie principal que el Estado autorizará a extraer ese año, la cual se define de acuerdo con criterios de conservación y sustentabilidad (Gelcich et al., 2010; Marín y Gelcich, 2012).

Si bien el sistema de AMERB puede considerarse exitoso como lo indicarían diversos estudios (Castilla et al., 2007; Gelcich et al., 2010; González et al., 2006; San Martín et al., 2010; entre otros), en especial si se compara con el sistema anterior y los sitios que actualmente se encuentran en libre acceso, ya sea desde el punto de vista ecológico, económico o social, es importante señalar que la implementación y operación del mismo no ha estado exenta de problemas (Aburto et al. 2013; Chávez et al., 2010; Stotz et al., 2006; Stotz et al., 2008; San Martín et al., 2010; entre otros). Al respecto, con la instauración del sistema de AMERB, las primeras solicitudes se centraron de preferencia en los sectores más atractivos para los pescadores ${ }^{6}$, reduciendo gradualmente la disponibilidad de las zonas libres o de libre acceso, y las que fueron quedando, además de ser sobreexplotadas, eran en general menos productivas y/o atractivas económicamente, lo que fue en desmedro de organizaciones de pescadores que se unieron al sistema más tarde (Stotz et al., 2006; Stotz et al., 2008). Esta situación produciría conflictos entre las organizaciones de pescadores que cuentan o no con estas áreas, o pescadores que no se encuentran organizados, ya que en general los pescadores que no forman parte del sistema se oponen a la exclusión de los usuarios de zonas que tradicionalmente consideraron de libre acceso con anterioridad a la instauración de este. ${ }^{7}$

Entre las consecuencias negativas que se han generado con la instauración del sistema, se destaca la actividad extractiva ilegal (Gelcich et al., 2005; González et al., 2006; Marín, 2010; Schumann, 2007; entre otros), lo cual se relaciona directamente con el aumento de los conflictos, tensión e incluso violencia (entre organizaciones de pescadores y/o sus miembros), afectando las decisiones relacionadas con el cumplimiento de la normativa vigente (Chávez y Palma, 2006). ${ }^{8}$

en cuyo objeto social incluyan la asesoría o la prestación de servicios para el desarrollo de proyectos de investigación en el ámbito de las ciencias del mar, debiendo contar al menos con una persona que posea título profesional o grado académico, en el área de biología, pesquería, acuicultura o ciencias del mar.

6 Con mayor producción y recursos comercialmente atractivos como el loco.

7 Gelcich et al. (2005) realizan una evaluación de la política de asignación de derechos de uso territorial analizando discursos de los propios pescadores, para entender los impactos y consecuencias de esta política, encontrando entre los pescadores algunas percepciones negativas hacia el sistema de AMERB, principalmente la existencia de lugares de acceso abierto (territorios históricos) cada vez más escasos y sobreexplotados, lo cual es utilizado como un medio para legitimar las reclamaciones para adaptarse a las condiciones generadas por la política y también reivindicar la cosecha ilegal entre las organizaciones de pescadores.

8 Otras consecuencias negativas de la instauración del sistema son la reducción del número de miembros de las organizaciones de gestión de las zonas económicamente menos 


\section{UN MODELO DE CUMPLIMIENTO INDIVIDUAL E HIPÓTESIS}

Con el propósito de examinar decisiones de cumplimiento de pescadores artesanales consideramos el modelo de un pescador individual neutral al riesgo, que opera en conjunto con otros pescadores en actividades de extracción de un recurso de propiedad común en un contexto de entrada cerrada. El análisis considera las siguientes situaciones: (i) ausencia de control regulatorio externo en términos de cuotas y fiscalización (vigilancia y sanciones); (ii) presencia de control externo, de parte de una autoridad oficial, en la forma de una cuota de extracción en conjunto con un nivel de vigilancia y sanciones para detectar y sancionar transgresiones.

\subsection{Recursos de propiedad común}

En el modelo que se presenta a continuación, los beneficios (y costos) que los individuos obtienen de la cosecha de un recurso depende del nivel de extracción $\left(x_{i}\right)$ y del stock disponible del recurso $(S)$. La función de beneficios individual está dada por:

$$
\pi_{i}=p x_{i}-\frac{\beta x_{i}^{2}}{2 S}+\alpha \sum_{j=1}^{n}\left(e_{j}-x_{j}\right) ; \quad i, j=1, \ldots, n .
$$

Donde $p$ representa el nivel de precio del recurso, $x_{i}$ es el nivel de cosecha individual, $S$ representa el nivel del stock del recurso, $\beta$ es un parámetro técnico asociado al costo de cosecha, $e_{i}$ es el nivel máximo factible que un pescador puede cosechar, y $\alpha$ representa el efecto de un cambio en la disponibilidad del recurso sobre el beneficio individual.

Considerando un número fijo de pescadores igual a $n$ y asumiendo que los mismos poseen dotaciones simétricas de $e_{i}$, esto es, $e_{i}=e$, podemos reescribir la función de pagos (1) de la siguiente forma:

$$
\pi_{i}=p x_{i}-\frac{\beta x_{i}^{2}}{2 S}+\alpha n e-\alpha \sum_{j=1}^{n} x_{j} ; \quad i, j=1, \ldots, n .
$$

Si un pescador $i$ elige $x_{i}$ para maximizar $\pi_{i}$, la condición de primer orden que caracteriza el nivel óptimo de extracción está dada por:

$$
\frac{\partial \pi_{i}}{\partial x_{i}}=p-\frac{\beta x_{i}}{S}-\alpha=0, \quad i=1, \ldots, n .
$$


Considerando un equilibrio de Nash de un juego simétrico, la condición anterior nos permite obtener el nivel de equilibrio de extracción, $x_{i}^{\text {nash }}$, el cual está dado por

$$
x_{i}^{\text {nash }}=\frac{S}{\beta}(p-\alpha) \text {. }
$$

Considerando $p>\alpha$, entonces $0 \leq x_{i} \leq e$.

Con el propósito de tener un patrón de comparación entre el comportamiento predicho a nivel individual en un equilibrio de Nash, caracterizamos a continuación una situación en que se seleccionan niveles de extracción individuales que maximizan el bienestar social o de grupo. Para optimizar el bienestar social del total de $n$ pescadores, maximizamos la suma de los pagos y calculamos la extracción socialmente óptima $x_{i}^{\text {social }}$, esto es,

$$
\max _{\left\{x_{1}, \ldots, x_{n}\right\}} W=\sum_{i=1}^{n} \pi_{i}=p \sum_{i=1}^{n} x_{i}-\frac{\beta}{2 S} \sum_{i=1}^{n} x_{i}^{2}+\alpha n^{2} e-\alpha n \sum_{i=1}^{n} x_{i} .
$$

La condición de primer orden es:

$$
\frac{\partial W}{\partial x_{i}}=p-\frac{\beta x_{i}}{S}-\alpha n=0, \quad i=1, \ldots, n .
$$

Lo cual define el nivel de extracción óptimo de acuerdo a la ecuación (5) a continuación,

$$
x_{i}^{\text {social }}=\frac{S}{\beta}(p-\alpha n)
$$

Considerando $p>\alpha$, entonces $0 \leq x_{i}^{\text {social }} \leq e$.

De lo anterior se desprende que $x_{i}^{\text {nash }}=\frac{S}{\beta}(p-\alpha)>x_{i}^{\text {social }}=\frac{S}{\beta}(p-\alpha n)$, lo que indica que el nivel de extracción socialmente óptimo será siempre menor al que resulta del equilibrio de Nash del juego no cooperativo.

\subsection{Cuota de extracción, vigilancia y sanciones para inducir cumplimiento}

Con el propósito de incorporar el efecto de una regulación externa, consideramos la introducción de un sistema de regulación formal sobre los pescadores que mantienen DUTP. La regulación consiste en el uso de los siguientes instrumentos: (a) una cuota global de extracción (y su correspondiente asignación a nivel individual), (b) actividad de vigilancia para detectar transgresiones de cuota, y (c) la imposición de una sanción económica (multa) en caso que una infracción sea detectada. Suponemos que el regulador determina la cuota en 
el nivel socialmente óptimo. Denotamos la probabilidad de detección $\theta$, y una multa por unidad de infracción constante e igual a $m$. Siempre que $m$ y $\theta$ sean positivos, la estrategia Nash de extracción en este contexto será menor que aquella que prevalece en ausencia de regulación. Incorporando lo anterior, podemos reescribir la función de pagos (2) de la siguiente forma:

$$
\pi_{i}=p x_{i}-\frac{\beta x_{i}^{2}}{2 S}+\alpha n e-\alpha \sum_{j=1}^{n} x j-\theta m\left(x_{i}-x_{i}^{\text {social }}\right) ; \quad i, j=1, \ldots, n .
$$

Para este caso la condición de primer orden que caracteriza el nivel óptimo de extracción es:

$$
\frac{\partial \pi_{i}}{\partial x_{i}}=p-\frac{\beta x_{i}}{S}-\alpha-\theta m=0 .
$$

Lo cual define el nivel de extracción de acuerdo con la ecuación (7) a continuación,

$$
x_{i}^{\text {nash_externa }}=\frac{S}{\beta}(p-\alpha-\theta m) .
$$

Considerando $p>\alpha+\theta m$, entonces $0 \leq x_{i}^{\text {nash_externa }} \leq e$.

Bajo el supuesto de neutralidad al riesgo, seleccionando apropiadamente $m$ y $\theta$, es posible para el regulador inducir estrategias de cosecha individuales consistentes con el nivel determinado previamente en un óptimo social.

Utilizando los resultados del modelo presentado previamente, podemos definir el nivel de desviación de la extracción bajo RPC respecto del nivel óptimo social de la siguiente forma:

$$
x_{i}^{\text {nash_rpc }}-x_{i}^{\text {social }}=\left(\frac{S}{\beta}\right)[\alpha(n-1)] .
$$

De igual forma, podemos definir el nivel de violación bajo regulación externa formal de la siguiente forma:

$$
\text { Violación }\left(r e g_{-} e x t\right)=x_{i}^{\text {nash_externa }}-x_{i}^{\text {social }}=\left(\frac{S}{\beta}\right)[\alpha(n-1)-\theta m] \text {. }
$$

Finalmente podemos comparar los niveles de extracción bajo RPC vs. el óptimo social, así como el nivel de la transgresión bajo regulación cuando la abundancia es alta vs. cuando la abundancia es baja. Como resultado de lo anterior, podemos escribir:

$$
\frac{\Delta\left(x_{i}^{\text {nash }-R P C}-x_{i}^{\text {social }}\right)}{\Delta S}=\left(\frac{1}{\beta}\right)[\alpha(n-1)]>0 .
$$




$$
\frac{\Delta \text { violación (regulaciónexterna })}{\Delta S}=\left(\frac{1}{\beta}\right)[\alpha(n-1)-\theta m]>0 .
$$

\subsection{Hipótesis}

Basados en los resultados del modelo previamente descrito, planteamos las siguientes hipótesis a evaluar:

Hipótesis 1: Los niveles de extracción individual son consistentes con el nivel predicho por el equilibrio de Nash tanto para el caso de recurso de propiedad común como en el caso de regulación formal (cuota, vigilancia y sanciones) en los escenarios de abundancia y escasez del recurso, respectivamente.

Hipótesis 2: En escenario de abundancia aumenta la brecha entre el nivel de extracción y el óptimo social, en comparación a una situación de escasez del recurso, en caso de RPC.

Hipótesis 3: En escenario de abundancia aumenta el nivel de infracción de cuotas de extracción en comparación a una situación de escasez del recurso, en caso de regulación formal.

\section{Diseño Experimental y Procedimientos}

En esta sección se presenta el diseño y procedimientos utilizados en los experimentos de campo aplicados a pescadores artesanales pertenecientes a organizaciones de pescadores que participan en el sistema de AMERB de la Región del Biobío, en la zona centro-sur de Chile.

\subsection{Aspectos de Diseño}

El experimento de campo fue diseñado basándose en el trabajo de Cárdenas et al. (2000) y posteriores aplicaciones como Murphy y Cárdenas (2004), este último implementado tanto con estudiantes como en el campo. Aplicaciones y adaptaciones posteriores de este diseño incluyen las realizadas por López (2004), Vélez et al. (2006), Vélez et al. (2010), y Maldonado y Moreno-Sánchez (2009) y Moreno-Sánchez y Maldonado (2010).

A continuación presentamos el diseño experimental utilizado, revisando los diferentes tratamientos utilizados, así como las funciones de pago utilizadas para calcular las ganancias obtenidas por los pescadores participantes de los experimentos. Los parámetros utilizados en el experimento se presentan en la Tabla 1. Con estos valores y usando las ecuaciones (3), (5) y (7) calculamos los resultados esperados para extracción y ganancias en cada caso. Estos resultados expresados en unidades de extracción y puntos experimentales se resumen en la Tabla 2.

Los resultados del modelo calibrado sugieren que en productividad biológica alta se espera un nivel de extracción de seis unidades para RPC y cuatro unidades para el caso de regulación externa formal. Del mismo modo, para el caso de productividad biológica baja se espera un nivel de extracción para RPC y regulación externa formal de tres y dos unidades respectivamente.

El nivel de extracción esperada sobre lo socialmente óptimo para el caso de RPC en escenario de abundancia es de cuatro unidades y el nivel de violación 
TABLA 1

VALORES DE LOS PARÁMETROS UTILIZADOS

\begin{tabular}{|lccccccc|}
\hline Parámetro & $p$ & $\beta$ & $\alpha$ & $e$ & $\theta$ & $m$ & $S$ \\
\hline Valor & 140 & 1600 & 20 & 8 & $1 / 5$ & 200 & 40,80 \\
\hline
\end{tabular}

Fuente: Elaboración propia basada en los experimentos realizados en este trabajo.

TABLA 2

NIVELES DE EXTRACCIÓN Y PUNTOS EXPERIMENTALES ESPERADOS POR RONDAS

\begin{tabular}{|lcccccc|}
\hline & \multicolumn{3}{c}{ Productividad Alta } & \multicolumn{3}{c|}{ Productividad Baja } \\
\cline { 2 - 7 } & \multicolumn{2}{c}{ Etapa 1 } & Etapa 2 & Etapa 1 & Etapa 2 \\
\hline Nivel de extracción & $x_{i}^{\text {nash }}$ & $x_{i}^{\text {social }}$ & $x_{i}^{\text {nash_Externa }}$ & $x_{i}^{\text {nash }}$ & $x_{i}^{\text {nash }}$ & $x_{i}^{\text {nash_Externa }}$ \\
& 6 & 2 & 4 & 3 & 1 & 2 \\
\hline Ganancia individual & 680 & 840 & 800 & 740 & 820 & 800 \\
Ganancia grupal & 3400 & 4200 & 4000 & 3700 & 4100 & 4000 \\
\hline
\end{tabular}

Fuente: Elaboración propia basada en los experimentos realizados en este trabajo.

esperada para regulación externa formal es de dos unidades. En tanto para el escenario de productividad biológica baja (escasez) se espera un nivel de extracción sobre lo socialmente óptimo de dos unidades para el caso de RPC y un nivel de violación de una unidad para regulación externa formal.

Los experimentos fueron diseñados sugiriendo a los sujetos el contexto específico del problema de extracción de recursos de uso común, enfrentados a la toma de decisiones sobre cómo aprovechar los recursos de que dependen los miembros del grupo, en este caso del recurso loco. Esta problemática es cercana a los sujetos experimentales, quienes se ven enfrentados a menudo, en su vida cotidiana, a transacciones de mercado, teniendo conocimiento y dominio de operaciones aritméticas básicas, además de verse enfrentados generalmente a incentivos de carácter económico.

\subsection{Procedimiento experimental}

En los experimentos cuyos resultados aquí se presentan participaron un total de 60 pescadores artesanales pertenecientes a organizaciones que operan bajo el régimen de Áreas de Manejo y Explotación de Recursos Bentónicos en la Región del Biobío. Los pescadores participantes fueron separados en 12 grupos de 5 personas. Los experimentos fueron aplicados entre diciembre de 2012 y febrero de 2013. En las sesiones experimentales se simuló la explotación conjunta de un recurso de propiedad común, similar a las actividades extractivas que realizan cotidianamente. Se realizaron dos tipos de sesiones experimentales compuestas cada una de dos etapas (20 rondas en total), la primera, denominada Línea Base 
RPC, fue repetida durante 10 rondas. En la segunda etapa se introdujo una cuota individual, además de vigilancia externa y la posibilidad de ser sancionado en el caso de ser sorprendido en incumplimiento de la norma.

En la Línea Base RPC se permitió la comunicación entre los miembros del grupo en la primera ronda; ello motivó la discusión del grupo durante un máximo de 5 minutos antes de tomar las decisiones de extracción, en los cuales no podían realizar acuerdos de transferencias de puntos o ganancias después de terminada la sesión. Cada individuo decidió cuánto extraer (entregando decisión al monitor, en tarjeta de decisión, quien calculará el total del grupo). El monitor anunciaba la extracción total del grupo con lo que cada individuo podía calcular sus puntos ganados en la ronda de acuerdo con la tabla de pagos entregada, correspondientes a baja o alta productividad biológica.

En la Fiscalización Formal se introdujo la regulación mediante el mecanismo de Derechos de Uso Territorial en la Pesca (DUTP), es decir, el sistema de AMERB, con cuotas individuales (no transferibles) al interior de la organización, además de vigilancia formal y sanción en el caso de ser sorprendidos incumpliendo. Al igual que en la Línea Base RPC se permitió la comunicación entre los miembros del grupo solo en la primera ronda de esta segunda etapa (ronda 11), durante un máximo de 5 minutos antes de tomar las decisiones de extracción, en los cuales no podían realizar acuerdos de transferencias de puntos o ganancias después de terminada la sesión. Se indicó la cuota individual y el monto de la sanción en caso de ser sorprendidos extrayendo sobre esta. Cada individuo decidió cuánto extraer, entregando su decisión al monitor quien calculaba el total del grupo. El monitor anuncia extracción total del grupo, con la que cada individuo podía calcular sus puntos ganados en la ronda de acuerdo con la tabla de pagos entregada, correspondientes a baja o alta productividad biológica. Cada pescador conocía la extracción de los miembros del grupo, lo cual era anunciado por el monitor, aunque se mantenía el anonimato. Posteriormente se realizaba la inspección a los pescadores del grupo de acuerdo con una probabilidad de monitoreo y sanción, imponiendo una multa por cada unidad por encima de la cuota individual fijada. El resultado de la inspección era anunciado públicamente (manteniendo el anonimato), indicando el número del sujeto inspeccionado y el resultado de la inspección.

En las sesiones se utilizaron set de materiales individuales que fueron entregados a cada participante con todo lo necesario para tomar sus decisiones. Estos set estaban compuestos por una Tabla de Pagos (igual para todos), lápices, Tarjetas de Decisión, Hojas de Decisiones, entre otros materiales. Además se utilizaron pizarras para transmitir las instrucciones. Se utilizó un ordenador portátil en el cual se introdujeron las decisiones de los pescadores para calcular sus ganancias.

En cada sesión participó un monitor acompañado de tres facilitadores. Al inicio, el monitor explicó de qué trataría la sesión experimental y cómo utilizar los materiales entregados. El monitor realizó rondas de ensayo para aclarar dudas y se realizaron preguntas de control para determinar si los pescadores estaban listos para comenzar.

Los grupos experimentales estaban compuestos por cinco miembros de la misma organización. Con la finalidad de evitar los efectos de presión del grupo, los sujetos participantes fueron distribuidos teniendo cuidado en separar los familiares, además se mantuvo el anonimato de los pescadores durante todas las rondas. Cada sesión experimental se extendió por aproximadamente dos horas y 
treinta minutos. Al finalizar la sesión experimental se llevó a cabo una encuesta para luego proceder a realizar los pagos en dinero en efectivo de manera privada.

\section{Resultados}

En esta sección presentamos los resultados de los experimentos de campo realizados con pescadores de la Región del Biobío. En la Tabla 3 se presenta un resumen de las características de los 60 pescadores participantes en los experimentos cuyos resultados son aquí analizados. Los sujetos experimentales fueron principalmente hombres (95\%), con una edad promedio de 45 años, con siete años de educación formal, en su mayoría casados (67\%) o conviviendo (12\%). El tamaño promedio del hogar de los pescadores participantes es de cuatro personas, en dichos hogares el pescador participante contribuye mayoritariamente con el ingreso principal del hogar ( $80 \%$ de los casos), con ingresos mensuales del grupo familiar en su mayoría en el rango de \$100.001-\$200.000 (42\%), seguido por el rango de ingresos menores o iguales a $\$ 100.000$ (32\%), cabe señalar que solo un $3 \%$ de los entrevistados indicó tener ingresos superiores a $\$ 401.000$. Respecto de la actividad pesquera principal, el $57 \%$ de los participantes en los experimentos mencionó ser buzo, el $37 \%$ pescador, $2 \%$ recolector de orilla, mientras el $5 \%$ señaló ser armador.

La Tabla 4 presenta estadística descriptiva referida a las ganancias obtenidas por los participantes en el experimento. Las ganancias individuales en el escenario de Productividad Biológica Baja alcanzaron un nivel promedio de \$12.382 y una desviación estándar de \$2.086. En tanto en el escenario de Productividad

TABLA 3

CARACTERÍSTICAS DE LOS PESCADORES

\begin{tabular}{|lrrr|}
\hline \multicolumn{1}{|c}{ Características } & Continental & Insular & Total \\
\hline Número de pescadores participantes & 35 & 25 & 60 \\
Promedio edad (años) & 44 & 46 & 45 \\
Promedio de años educación formal & 8 & 7 & 7 \\
\% Hombres & 91 & 100 & 95 \\
\% Mujeres & 9 & 0 & 5 \\
\% Pescadores & 34 & 40 & 37 \\
\% Buzos & 57 & 56 & 57 \\
\% Recolectores de Orilla & 3 & 0 & 2 \\
\% Armadores & 6 & 4 & 5 \\
\% Realiza actividades no pesqueras (económica) & 14 & 24 & 18 \\
\% Principal fuente de ingreso del hogar & 71 & 92 & 80 \\
Promedio integrantes hogar & 4 & 4 & 4 \\
\% Rango ingresos Menos o igual a \$ 100.000 & 34 & 28 & 32 \\
\% Rango ingresos entre \$ 100.001 y \$ 200.000 & 43 & 40 & 42 \\
\% Rango ingresos entre \$ 200.001 y \$ 300.000 & 17 & 28 & 22 \\
\% Rango ingresos entre \$ 300.001 y \$ 400.000 & 3 & 0 & 2 \\
\% Ingreso superior a \$ 400.001 & 3 & 4 & 3 \\
\hline
\end{tabular}

Fuente: Elaboración propia basada en las encuestas realizadas en este trabajo. 
TABLA 4

GANANCIAS INDIVIDUALES SEGÚN TRATAMIENTO (20 RONDAS). (CIFRAS EN PESOS CHILENOS)

\begin{tabular}{|lccccc|}
\hline \multicolumn{5}{c|}{ Estadística Descriptiva } \\
\hline & $\mathrm{N}$ & Mínimo & Máximo & Media & Desviación estándar \\
\hline Escasez & 30 & 6.338 & 15.554 & $12.382,40$ & $2.085,566$ \\
Abundancia & 30 & 13.421 & 17.129 & $15.333,50$ & 907,214 \\
\hline
\end{tabular}

Fuente: Elaboración propia basada en los experimentos realizados en este trabajo.

Biológica Alta, las ganancias promedio por individuo participante alcanzaron a \$ 15.554, con una desviación estándar de \$ 907.

Estudiamos a continuación los promedios de las decisiones de extracción de los pescadores artesanales participantes en el experimento. Específicamente, a continuación se presenta un resumen de las decisiones promedio de los individuos, divididos según la productividad biológica aplicada (ver Gráficos 1 y 2).

Los datos referidos a resultados obtenidos bajo Productividad Biológica Alta (ver Gráfico 1) sugieren que durante las primeras 10 rondas, las decisiones de extracción promedio comenzaron en 4 unidades produciéndose un incremento en el nivel de extracción en el transcurso de nuevas rondas, llegando a cerca de 5,5 unidades. Por su parte las extracciones con Productividad Biológica Baja (ver Gráfico 2) comenzaron más altas que en la Productividad Biológica Alta, sobre las 5 unidades promedio, para luego comenzar a disminuir paulatinamente estabilizándose entre la ronda 7 y 10, alcanzando un promedio cercano a 3,5 unidades.

Respecto del nivel de cosecha, en ambos casos (Productividad Biológica Alta y Productividad Biológica Baja para las primeras 10 rondas) se observa que estas difieren del nivel predicho por el equilibrio de Nash (correspondiente a 6 unidades con Productividad Alta y 3 unidades con Productividad Baja), situándose por debajo del equilibrio de Nash en el caso de los tratamientos aplicados con Productividad Biológica Alta, con un promedio de extracción de aproximadamente 5 unidades, es decir, cerca de 1 unidad bajo el equilibrio de Nash (ver Tabla 5). En el caso del tratamiento aplicado con Productividad Biológica Baja las extracciones se situaron sobre el equilibrio de Nash, con un promedio de extracción de 4 unidades aproximadamente, es decir, cerca de 1 unidad sobre este (ver Tabla 5).

El nivel de desviación de la extracción bajo RPC respecto del nivel óptimo social (el que obtenemos utilizando la ecuación 8), en el caso de Productividad Biológica Alta se situó bajo la desviación esperada (correspondiente a 4 unidades). Específicamente, el nivel de desviación promedio alcanzó en este caso a 2,75 unidades de cosecha sobre el óptimo social. Respecto del caso de Productividad Biológica Baja, el nivel de desviación bajo RPC se situó sobre la desviación esperada (correspondiente a 2 unidades), con 2,03 unidades de cosecha sobre el óptimo social. 
TABLA 5

TEST NO PARAMÉTRICO EXTRACCIÓN TEÓRICA VS EXTRACCIÓN OBSERVADA, WILCOXON-MANN-WHITNEY

\begin{tabular}{|llccc|}
\hline Comparación & Tratamientos & $\begin{array}{c}\text { Extracción } \\
\text { Teoría }\end{array}$ & $\begin{array}{c}\text { Extracción } \\
\text { Promedio }\end{array}$ & $p$-value \\
\hline \multirow{2}{*}{ Abundancia } & Línea Base RPC & 6 & 4,75 & $\begin{array}{c}0,000 \\
(9,700)\end{array}$ \\
\cline { 2 - 5 } & Formal & 4 & 3,04 & $\begin{array}{c}0,000 \\
(11,775)\end{array}$ \\
\hline \multirow{2}{*}{ Escasez } & Línea Base RPC & 3 & 4,03 & $\begin{array}{c}0,000 \\
(-6,695)\end{array}$ \\
\cline { 2 - 5 } & Formal & 2 & 2,65 & $\begin{array}{c}0,1132 \\
(1,584)\end{array}$ \\
\hline
\end{tabular}

Fuente: Elaboración propia basada en los experimentos realizados en este trabajo. Valor z entre paréntesis.

En el caso de los resultados para la segunda etapa (rondas 11-20) en la cual se introdujo el tratamiento de control formal (cuota y fiscalización exógena), las extracciones promedio tuvieron un fuerte descenso en comparación con las primeras 10 rondas, tanto para los casos con Productividad Biológica Alta como Productividad Biológica Baja (ver Gráfico 2 y Tabla 5).

Respecto del nivel de cosecha en la segunda etapa, en el escenario de Productividad Biológica Alta, el referido nivel en el caso de tratamiento Formal alcanzó un promedio de 3 unidades aproximadamente (ver Tabla 5), es decir, 1 unidad aproximadamente bajo el nivel predicho por el equilibrio de Nash, correspondiente a 4 unidades, y cerca de 1 unidad sobre la cuota fijada por el regulador (cuota individual $=2$ ). En el escenario con Productividad Biológica Baja, el nivel de extracción en el tratamiento Formal alcanzó un promedio de 3 unidades aproximadamente para la segunda etapa (ver Tabla 5), es decir, cerca de 1 unidad sobre el nivel predicho por el equilibrio de Nash, correspondiente a 2 unidades y 2 unidades sobre la cuota fijada por el regulador (cuota individual $=1$ ).

Finalmente respecto del nivel de infracción de cuotas en el caso de la regulación formal (que obtenemos utilizando la ecuación 9), tenemos que en el escenario de Productividad Biológica Alta el referido nivel se encuentra bajo el esperado (correspondiente a 2 unidades), con 1,04 unidades sobre la cuota fijada por el regulador. En tanto en el caso de Productividad Biológica Baja, el nivel de infracción se encuentra sobre el nivel de infracción esperado (correspondiente a 1 unidad) con 1,65 unidades de cosecha sobre la cuota fijada por el regulador.

La primera hipótesis de este estudio plantea que los niveles de extracción individual son consistentes con el nivel predicho por el equilibrio de Nash tanto para el caso de recurso de propiedad común como en el caso de regulación formal en escenarios de abundancia y escasez del recurso loco. Nuestros resultados indican que los niveles de extracción individual no son consistentes con el nivel predicho por el equilibrio de Nash, existiendo diferencias estadísticamente significativas entre los valores de la extracción teórica (predicha) y la extracción observada, salvo en el caso del tratamiento formal aplicado en el escenario de 


\section{GRÁFICO 1 \\ EVOLUCIÓN DE LAS EXTRACCIONES PROMEDIO PARA CADA TRATAMIENTO CON ALTA PRODUCTIVIDAD BIOLÓGICA (ABUNDANCIA)}

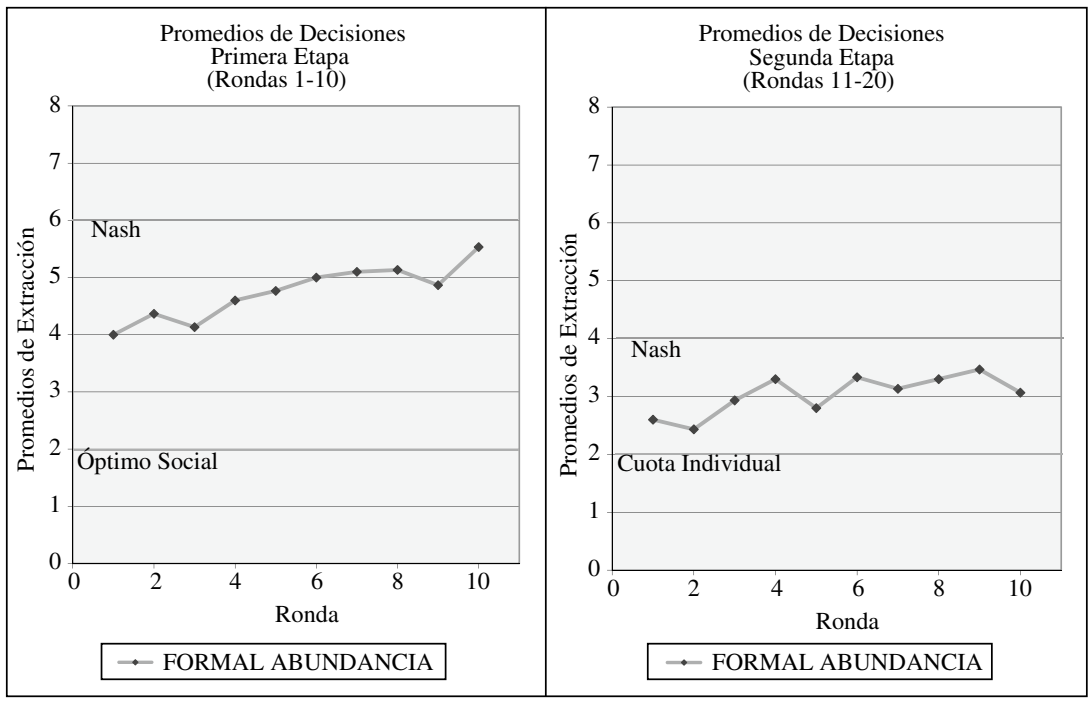

Fuente: Elaboración propia basada en los experimentos realizados en este trabajo.

\section{GRÁFICO 2}

EVOLUCIÓN DE LAS EXTRACCIONES PROMEDIO PARA CADA TRATAMIENTO CON BAJA PRODUCTIVIDAD BIOLÓGICA (ESCASEZ)

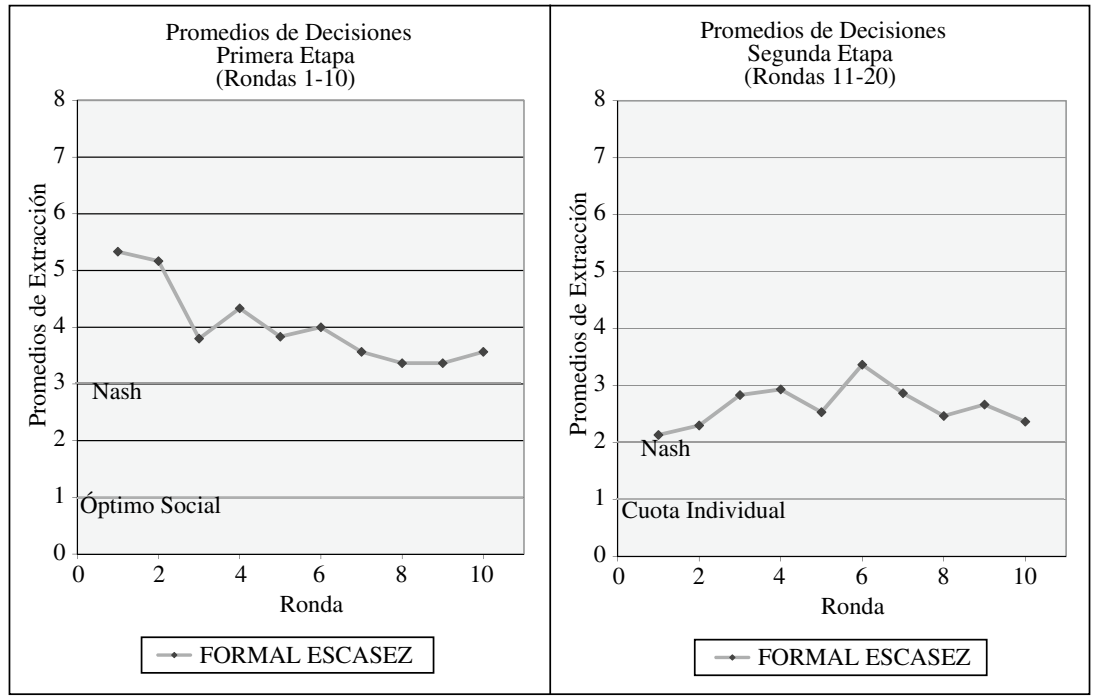

Fuente: Elaboración propia basada en los experimentos realizados en este trabajo. 
TABLA 6

TEST NO PARAMÉTRICO RPC Y FORMAL, WILCOXON-MANN-WHITNEY

\begin{tabular}{|lcc|}
\hline \multicolumn{1}{|c}{ Tratamientos } & Magnitud Diferencia & p-value \\
\hline RPC Abundancia & 2,75 & 0,1692 \\
& & $(-1,375)$ \\
\hline RPC Escasez & 3,03 & 0.0001 \\
\cline { 1 - 2 } Formal Abundancia & 1,04 & $(-3.866)$ \\
\hline Formal Escasez & 1,65 & \\
\hline
\end{tabular}

Fuente: Elaboración propia basada en los experimentos realizados en este trabajo.

Valor $z$ entre paréntesis.

escasez (ver Test No Paramétrico Wilcoxon-Mann-Whitney, Tabla 5). Presentando extracciones bajo el equilibrio de Nash en el caso de escenario de abundancia y sobre el equilibrio de Nash en el caso de escasez.

La segunda hipótesis del estudio plantea que en el escenario de abundancia aumenta la brecha entre el nivel de extracción y el óptimo social, en comparación a una situación de escasez del recurso, en caso de RPC. Nuestros resultados indican que la brecha entre el nivel de extracción y el óptimo social no aumenta en el escenario de abundancia, sino que esto ocurre en el escenario de escasez. No obstante, la diferencia entre los niveles de extracción de ambos escenarios en RPC no es significativa (ver Test No Paramétrico Wilcoxon-Mann-Whitney, Tabla 6).

Finalmente la tercera hipótesis del estudio plantea que en el escenario de abundancia aumenta el nivel de infracción de cuotas de extracción en comparación a una situación de escasez del recurso, en caso de regulación formal. Nuestros resultados indican que el nivel de infracción de cuotas no aumenta en el escenario de abundancia. Sin embargo, al igual que en la hipótesis anterior, el nivel de infracción aumenta en el escenario de escasez, presentando una diferencia estadísticamente significativa entre ambos escenarios en regulación formal (ver Test No Paramétrico Wilcoxon-Mann-Whitney, Tabla 6) ${ }^{9}$.

Los resultados de este trabajo son consistentes con la relación entre la cooperación y productividad de un sistema natural propuesta por Ostrom (2009), en la cual si el recurso está cerca de agotarse, los usuarios no percibirían la necesidad de manejo para el futuro, es decir, no percibirían beneficios asociados a la cooperación. En este contexto, los individuos seguirían estrategias egoístas, en este caso, un comportamiento infractor. Un resultado similar presentan Blanco et al. (2011), encontrando que en escenarios de reducción significativa en los niveles

9 Cabe señalar que adicionalmente los test no paramétricos fueron realizados excluyendo solo las dos primeras rondas de cada etapa, y excluyendo las dos primeras rondas y las dos últimas rondas de cada etapa; sin embargo, no se presentaron diferencias en los resultados de los test. 
de abundancia existe un riesgo de agotamiento de estos, porque los individuos responden de manera más egoísta mediante el aumento de sus extracciones ${ }^{10}$.

\section{Conclusiones}

En este artículo presentamos los resultados de la aplicación de experimentos de campo diseñados con el propósito de estudiar el efecto de diferentes niveles de abundancia de un recurso natural sobre las decisiones de extracción y transgresión bajo diferentes sistemas de regulación.

Los resultados principales obtenidos son los siguientes. Primero los niveles de cosecha tanto en el caso de abundancia como escasez tienen una diferencia significativa respecto del equilibrio de Nash predicho, aunque se acercan más a este que al óptimo social, bajo RPC y bajo regulación externa. El nivel de cosecha se encuentra bajo el equilibrio de Nash en el caso de abundancia, en RPC como en regulación externa, y sobre el equilibrio de Nash en el caso de escasez, tanto en RPC como en regulación externa. Segundo, la mayor desviación de la extracción bajo RPC respecto del óptimo social se presenta en el escenario de escasez. Tercero, el mayor nivel de infracción de cuotas de extracción en el caso de la regulación formal se presenta en el escenario de escasez.

En los escenarios con abundancia, los pescadores se comportaron de una manera más cooperativa, es decir, realizan menores niveles de extracción e infracción que en los escenarios bajo escasez.

De acuerdo con nuestros resultados, el comportamiento de los pescadores enfrentados a escenarios de abundancia y escasez presenta diferencias significativas. En situaciones de abundancia, cuando se introduce control de cuota y fiscalización exógena, los pescadores disminuyen sus extracciones respecto de la línea base RPC, presentando menores niveles de extracción e incumplimiento, mientras que en situación de escasez, si bien los pescadores también disminuyen sus extracciones respecto de la línea Base RPC, presentan niveles de incumplimiento y extracciones más altas que en el escenario de abundancia, incluso con extracciones sobre el equilibrio de Nash.

Estos resultados son relevantes especialmente si se considera que para las organizaciones que enfrentan una baja productividad biológica en sus áreas de manejo, los costos que implican la autoorganización y el tiempo destinado a estas, por ejemplo, a la aplicación de sus sistemas de vigilancia y fiscalización (cuando los tienen) pueden ser demasiado altos. En este contexto existe el peligro de que no se generen los suficientes beneficios esperados que promuevan e incentiven su cuidado, evitando la sobreexplotación. Al respecto, en el país existen muchas organizaciones que presentan áreas con baja productividad biológica, aunque no se sabe su número exacto ni las magnitudes de esta baja productividad (no existen criterios claros para definir que un área presenta escasez, abundancia

10 El trabajo de Blanco et al. (2011) explora las respuestas de comportamiento de agentes socioeconómicos a los cambios exógenos en la disponibilidad de recursos. Los sujetos en su experimento eran usuarios rurales de agua, en Colombia, que participaron en un experimento de campo (RPC) en el que los niveles de los recursos variaban a lo largo de las diferentes etapas del juego. 
media, abundancia alta), además muchas de ellas presentan este escenario desde el inicio de la asignación de derechos, o bien durante el transcurso de los años han disminuido su productividad, debido a shocks endógenos o exógenos (por ejemplo, debido a la sobreexplotación de agentes internos y/o externos, cambios ambientales, impactos por efecto de actividades industriales, entre otros).

Respecto de este último punto (shocks endógenos y exógenos), se pone en relevancia la sensibilidad de estos sistemas de administración, como sistemas socioecológicos, en los cuales la relación ecológica y social va de la mano para lograr el éxito, ya que existe la posibilidad de que organizaciones que cuentan con áreas relativamente productivas y aparentemente sólidas en lo organizacional, enfrentadas a shocks que reduzcan demasiado rápido la abundancia de recursos, no puedan adaptarse a estos cambios, o bien un cambio en la dirección de las organizaciones puede llevar a la sobreexplotación y el desmoronamiento de la organización.

Por estas razones cobra relevancia que las organizaciones cuenten con indicadores que den cuenta no solo de las condiciones ecológicas del recurso (abundancia), sino también que evalúen el estado de las organizaciones (planes de manejo, autoorganización, sistemas de vigilancia internos y del Estado, entre otros), que permitan establecer posibles acciones conjuntas (Estado-organización), por ejemplo, el establecer una mayor presencia de los organismos encargados de la fiscalización externa (Armada, Sernapesca) en coordinación con las propias organizaciones en el caso de existir niveles de abundancia demasiado bajos, que no demanden solamente el uso interno de recursos para asumir estas labores de parte de las organizaciones. Tales indicadores debieran ser incluidos en los estudios de seguimiento anuales, existiendo una permanente evaluación de estos e inclusión en el diseño regulador de parte del Estado y las propias organizaciones. La inclusión de tales indicadores permitirían la adecuada definición de posibles líneas de acción y el desarrollo de proyectos que fortalezcan el sistema, mediante iniciativas como el fortalecimiento organizacional, proyectos de repoblamiento, acuicultura de pequeña escala, entre otros, que permita, de acuerdo con sus propias necesidades, prevenir la disminución de recursos, recuperarlos, o bien una adaptación más rápida frente a condiciones adversas.

En definitiva nuestro trabajo pone de manifiesto el cuidado que debe tenerse para desarrollar un adecuado diseño e implementación de sistemas de derechos de uso territorial cuando se está en presencia de heterogeneidad espacial en términos de productividad biológica.

\section{REFERENCIAS}

Aburto, J.; Gallardo, G.; Stotz, W.; Cerda, C.; Mondaca-Schachermayer, C. y Vera, K. (2013). "Territorial user rights for artisanal fisheries in Chile - intended and unintended outcomes", Ocean \& Coastal Management, Vol. 71, pp. 284-295. doi:10.1016/j.ocecoaman.2012.09.015

Blanco, E., Lopez, M.C., y Villamayor-Tomás, S. (2011). "Does water scarcity lead to overuse? Evidence from field experiments", Working paper W11-15. Bloomington: Indiana University, Workshop in Political Theory and Policy Analysis. 
Cárdenas, J.C.; Strandlund, J. y Willis, Cleve (2000). "Local Environmental Control and Institutional Crowding-out", World Development, Vol. 28, $\mathrm{N}^{\mathrm{o}} 10$, pp. 1719-1733.

Cárdenas, J.C. (2009). "Dilemas de lo colectivo. Instituciones pobreza y cooperación en el manejo local de los recursos de uso común", Bogotá, Universidad de los Andes, Facultad de Economía, CEDE, Ediciones Uniandes, p. 336.

Castilla, J.C.; Gelcich, S. y Defeo, O. (2007). "Successes, lessons, and projections from experience in marine benthic invertebrate artisanal fisheries in Chile". In: McClanahan, T.R.; Castilla, J.C. (Eds.), Fisheries Management: Progress Towards Sustainability. Blackwell, New Delhi, pp. 25-42.

Castilla, J.C. (2010). "Fisheries in Chile: small pelagics, management, rights, and sea zoning", Bulletin of Marine Science, Vol. 86, № 2, pp. 221-234.

Chávez, C. y Palma, M.(2006). "Normas y Cumplimiento en Áreas de Manejo de Recursos Bentónicos Estudio de Caso en la Región del Bío-Bío". Estudios Públicos, Santiago, No 103, pp. 237-276.

Chávez, C.; Dresdner, J.; Quiroga, M.; Baquedano, M.; González, N.; Castro, R. (2010). "Evaluación Socio-Económica de la Pesquería del Recurso Loco Asociada al Régimen de Áreas de Manejo, como Elemento de Decisión para la Administración Pesquera", Proyecto FIP 2008-31.

Gelcich, S.; Hughesb, T.; Olsson, P.; Folke, C.; Defeo, O.; Fernández, M.; Foale, S.; Gunderson, L.; Rodríguez-Sickerth, C.; Schefferi, M.; Steneckj, R. y Castilla J.C. (2010). "Navigating transformations in governance of Chilean marine coastal resources", Proceedings of the National Academy of Sciences of the United States of America, Vol. 107, No 39, pp. 1679416799. http://www.pnas.org/cgi/doi/10.1073/pnas.1012021107

Gelcich, S.; Edwards-Jones, G.; Kaiser, M.J. y Castilla, J.C. (2006). "Comanagement policy can reduce resilience in traditionally managed marine ecosystems", Ecosystems, Vol. 9, № 6, pp. 951-966.

Gelcich, S.; Edwards-Jones, G.; Kaiser, M.J. y Watson, E. (2005). "Using Discourses for Policy Evaluation: The Case of Marine Common Property Rights in Chile", Society \& Natural Resources, Vol. 18, № 4, pp. 377-391.

González, E. (1996). "Territorial use rights in Chilean fisheries", Marine Resource Economics, Vol. 11, pp. 211-218

González, J.; Stotz, W.; Garrido, J.; Orensanz, J.M.; Parma, A.M.; Tapia, C.; Zuleta, A., (2006). "The Chilean TURF system: how is it performing in the case of the loco fishery?", Bulletin of Marine Science, Vol. 78, № 3 , pp. 499-527.

Harrison, G.W. y List, J.A. (2004). "Field Experiments", Journal of Economic Literature, Vol. 42, $\mathrm{N}^{\mathrm{o}}$ 4, pp. 1009-1055.

López, M. (2004). "Experimental Economics and Participative Methodologies: Looking for Theoretical and Methodological bridges to Analyse Collective Action in Common-Use Resources Management". Paper presentado en "Conference on experiences of combining qualitative and quantitative methods in poverty appraisal" Toronto. http://www.utoronto.ca/mcis/ q2/index.htm

Maldonado, J.H. y Moreno-Sánchez, R.P. (2009). "Does Scarcity Exacerbate the Tragedy of the Commons? Evidence from Fishers' Experimental 
Responses”, Documento CEDE 2009-22, Uniandes, Bogotá, October 2009. http://ssrn.com/abstract=1502899 http://dx.doi.org/10.2139/ssrn.1502899

Marín, A. y Berkes, F. (2010). "Network approach for understanding small-scale fisheries governance: The case of the Chilean coastal co-management system", Marine Policy, Vol. 34, No 5, pp. 851-858.

Moreno-Sánchez, R.P. y Maldonado, J.H. (2010). "Evaluating the role of co-management in improving governance of marine protected areas: An experimental approach in the Colombian Caribbean”, Ecological Economics, Elsevier, Vol. 69, № 12, pp. 2557-2567.

Murphy, J. y Cardenas, J.C., (2004). “An Experiment on Enforcement Strategies for Managing a Local Environmental Resource”, Journal of Economic Education, Vol. 35, № 1, pp. 47-61.

Osés-Eraso, N.; Udina, F. y Viladrich-Grau, M. (2008). "Environmental versus Human-Induced Scarcity in the Commons: Do They Trigger the Same Response?", Environmental and Resource Economics, Vol. 40, № 4, pp. 529-550.

Osés-Eraso, N. y Viladrich-Grau, M.(2007). "Appropriation and concern for resource scarcity in the commons: An experimental study", Ecological Economics, Vol. 63, No 2-3, pp. 435-445.

Ostrom, E. (2009). "A general framework for analyzing sustainability of social-ecological systems", Science (New York, N.Y.), Vol. 325, № 5939, pp. 419-22.doi:10.1126/science.1172133

San Martín, G.; Parma, A.M. y Orensanz, J.M.L. (2010). “The Chilean experience with territorial use rights in fisheries”. In: Grafton, R.Q.; Hilborn, R.; Squires, D.; Tait, M.; Williams, M. (Eds.), Handbook of Marine Fisheries Conservation and Management. Oxford University Press, Inc., New York, pp. 324-337.

Schumann, S. (2007). “Co-management and 'consciousness': Fishers' assimilation of management principles in Chile", Marine Policy, Vol. 3, $\mathrm{N}^{\mathrm{o}}$ 2, pp. 101-111.

Stotz, W.; Aburto, J.; Caillaux, L.; Lancellotti, D.; Valdebenito, M.; Tapia, C.; Guajardo, P. y Araya, P. (2006). "Diseño de plan piloto de ordenamiento espacial de pesquerías bentónicas en la IV región, Chile”. Informe Final Proyecto FIP 2003-15. Universidad Católica del Norte. Facultad de Ciencias del Mar p. 107.

Stotz, W.; Caillaux, L.; Cecchi, D.F.; Escobar, M.; Garay, R.; Lancellotti, D.; Valdebenito, J.M. y Zuñiga, S. (2008). "Evaluación del proceso de implementación de la medida de administración áreas de manejo y explotación de recursos bentónicos (AMERB) en las regiones III y IV y la elaboración de una propuesta de mejoramiento de la medida", Proyecto FIP 2005- 34 Grupo de Ecología y Manejo de Recursos, Facultad de Ciencias del Mar, Universidad Católica del Norte, Coquimbo, 424 pp.

Subsecretaría de Pesca (2013). "Estado Situación Actual Âreas de Manejo por Región y Nacional”. http://www.subpesca.cl/controls/neochannels/ neo_ch847/neochn847.aspx?appinstanceid=855\&pubid $=1662$

Subsecretaría de Pesca (2000). “Áreas de Manejo y Explotación de Recursos Bentónicos", Documento de Difusión No 1; Junio.

Vélez, M.A.; Stranlund, J.K. y Murphy, J.J. (2006). "Within and Between Group Variation of Individual Strategies in Common Pool Resources: Evidence 
from Field Experiments", Working Paper, Department of Resource Economics, University of Massachusetts, Amherst.

Vélez, M.A., Stranlund, J.K. y Murphy, J.J. (2010), “Centralized and decentralized management of local common resources in the developing world: experimental evidence from fishing communities in Colombia", Economic Inquiry, 48 (2), 254-265.

Villena, M. y Chávez, C. (2005). "On the Enforcement of Territorial Use Rights Regulations: A Game Theoretic Approach", Economia, Vol. 6, No 1, pp. 1-44.

Wilen, J.; Cancino, J. y Uchida. H. (2012). "The Economics of Territorial Use Rights Fisheries, or TURFs", Review of Environmental Economics and Policy, Vol. 6, N 2, pp. 237-257. 


\section{ANEXO 1}

\section{INSTRUCCIONES $^{11}$}

El monitor y facilitadores deberán entregar las instrucciones y materiales (ver anexo 1, materiales experimentos), además de colocar un resumen de las instrucciones junto a la tasa de cambio en un lugar visible.

Muchas gracias por participar en esta investigación sobre el manejo de recursos naturales, en el cual su presencia es clave, ya que son ustedes quienes tienen experiencia directa con las Áreas de Manejo y Explotación de Recursos Bentónicos (AMERB) en la Región.

Durante la jornada de hoy les presentaremos una forma diferente de participar en investigaciones, en donde cada uno de ustedes tendrá un papel fundamental, activo y dinámico, que esperamos sea de su agrado y muy interesante para ustedes. En términos generales este estudio se interesa en las decisiones de carácter económico que toman las personas, por lo cual durante la sesión, ustedes deberán tomar decisiones económicas que les permitirán ganar una cantidad de dinero en efectivo, por lo cual es de suma importancia que presten mucha atención a estas instrucciones. Es importante señalar que los fondos que cubren todos los gastos de esta investigación son otorgados por el Programa Latinoamericano y del Caribe de Capacitación en Economía Ambiental - LACEEP.

Si se preguntan por qué usamos dinero para la toma de decisiones, la respuesta es que lo usamos porque necesitamos que las personas tomen decisiones de tipo económico; es decir, que tomen decisiones que tengan consecuencias para su bolsillo, tal como sucede en la vida diaria.

\section{INTRODUCCIÓN}

Durante la jornada les presentaremos una situación en la que ustedes serán parte de una organización de pescadores, debiendo tomar decisiones sobre cómo aprovechar los recursos naturales de que depende la organización, en este caso del recurso loco.

Usted formará parte de un grupo de cinco (5) pescadores que forman parte de su organización en la realidad. En caso que hayan escuchado comentarios de otros grupos que participaron en alguna actividad similar, las decisiones que deberán tomar durante la jornada de hoy son diferentes, por lo cual esos comentarios no son aplicables a las decisiones que deberán tomar hoy.

Durante la toma de decisiones hablaremos de varias rondas en que deberán decidir. Estas rondas son equivalentes, por ejemplo, a años o temporadas de cosecha del recurso loco. Al final de la toma de decisiones, usted ganará una cantidad de puntos convertibles en dinero real, que dependerán de las decisiones que tome durante estas rondas y que deberán ser multiplicadas por 0,9 (tasa de cambio), procedimiento que explicaremos en detalle más adelante.

11 A continuación se presentan las instrucciones utilizadas en el caso de abundancia. Instrucciones adaptadas de Cárdenas, 2009. 
Para comenzar y poder tomar las decisiones, usted recibirá a continuación un sobre en el que se incluyen los siguientes elementos que explicaremos a continuación:

\section{a. Consentimiento informado}

Usted ha recibido una hoja de consentimiento informado. Esta hoja es un requisito para las universidades que hacen estudios en donde participan personas. En esta hoja les informamos a ustedes acerca de la confidencialidad y manejo de la información que recogemos en esta actividad, y ustedes firman, si aceptan participar, certificando que fueron informados del proyecto y del manejo de dicha información. Por favor lea esta hoja antes de firmarla (Ver Anexo 1, materiales experimentos letra a).

\section{b. Tarjeta con número de identificación}

Recibirá una tarjeta con un número de identificación que deberá guardar, ya que los pagos finales se realizarán previa presentación de este número (ver Anexo 1, materiales experimentos letra b). Puede observar que en el número de identificación aparecen dos ítems, el primero indica su participación en la totalidad del experimento, el cual será timbrado al finalizar esa actividad, el segundo ítem indica su participación en la encuesta que será timbrado una vez finalizada. Es muy importante que guarde esta tarjeta, ya que los pagos se realizarán previa entrega de esta tarjeta, la cual debe contener los dos timbres, descritos anteriormente, que aseguren que usted participó en ambas actividades, toma de decisiones y encuesta, y que las completó en su totalidad. Solo quienes participen en ambas actividades podrán cobrar los pagos en dinero efectivo de acuerdo con su toma de decisiones.

\section{c. Tabla de pagos}

La TABLA DE PAGOS tiene toda la información que se necesita para tomar su decisión en cada ronda de la actividad (ver Anexo 1, materiales experimentos letra c). Los números que aparecen dentro de la tabla corresponden a puntos, que son convertibles a pesos chilenos, que usted puede ganar en cada ronda, dependiendo de su decisión y la decisión de los demás integrantes del grupo.

Al finalizar las rondas de toma de decisiones, sumaremos el total de puntos ganados y los convertiremos a pesos chilenos que serán entregados personalmente a cada uno de los participantes en dinero efectivo, una vez que cumplan con todas las indicaciones descritas anteriormente.

Inicialmente, en cada ronda, lo único que ustedes tienen que decidir es el NIVEL DE EXTRACCIÓN DE LOCO que desea efectuar, escogiendo una columna entre 1 y 8 , cada unidad corresponderá a una centena de locos, es decir, 100 locos, por lo cual si usted elige 3 centenas, esto corresponderá a la extracción de 300 locos. Sin embargo, deberá considerar que los puntos que usted ganará personalmente, dependen no solo de MI NIVEL DE EXTRACCIÓN DE LOCOS (indicados por las columnas de la tabla), sino también del NIVEL DE EXTRACCIÓN DE LOCOS DE LOS OTROS, es decir, las cuatro perso- 
nas restantes del grupo, indicadas en las filas de la tabla. No se preocupe, más adelante explicáremos en detalle cómo se ganan puntos con varios ejemplos.

\section{d. Tarjetas de decisión}

En las TARJETAS DE DECISIÓN usted observará un número que será igual al indicado en la tarjeta de identificación (ver Anexo 1, materiales experimentos letra d). Por favor, revise que todas las TARJETAS DE DECISIÓN tengan el mismo número.

En cada ronda, ustedes van a escribir su decisión de manera individual y privada, escogiendo un número entre 1 centena (100 locos) y 8 centenas ( 800 locos), en una TARJETA DE DECISIÓN, el cual representará las unidades del recurso loco que usted ha decidido extraer en la ronda. Es muy importante para esta actividad que los números escritos sean mantenidos en privado, es decir, no sean mostrados ni comentados a los demás miembros del grupo.

\section{e. Hoja de decisiones}

La HOJA DE DECISIONES le permitirá saber cuánto ha ganado en cada ronda (ver Anexo 1, materiales experimentos letra e). Para saber cuántos puntos ganará en cada ronda, usted deberá saber la decisión que tomaron los demás integrantes de manera grupal. Para esto, el monitor recogerá en cada una de las rondas las cinco tarjetas correspondientes a su grupo, encargándose de sumar el total de NIVEL DE EXTRACCIÓN DE LOCOS que el grupo decidió extraer en esa ronda. Luego el monitor anunciará el total del grupo, momento en el cual cada uno de ustedes podrá calcular los puntos que ganó en la ronda usando la HOJA DE DECISIONES.

A continuación vamos a explicar en detalle cómo se usa esta hoja, con unos ejemplos, para lo cual utilizaremos las primeras filas que están marcadas como "Práctica".

\section{Ejemplo 1 (tabla de pagos alta)}

Supongamos que durante una ronda usted decidió extraer 5 centenas (500) unidades de loco. En primer lugar usted deberá escribir en la TARJETA DE DECISIÓN el número 5 (equivalente a 500 unidades de loco). También debe escribir este número en la Columna A (primera columna) de la HOJA DE DECISIONES.

El monitor recogerá las cinco tarjetas y procederá a calcular el total del grupo. Supongamos que el grupo en total sumó 25 centenas ( 2500 unidades de loco). Entonces escribimos 25 en la Columna B (segunda columna) de la HOJA DE DECISIONES.

Para calcular la Columna C (tercera columna), restamos MI NIVEL DE EXTRACCIÓN DE LOCOS (Columna A) al total del grupo (Columna B), y así obtenemos el NIVEL DE EXTRACCIÓN DE LOCOS DE LOS OTROS que escribimos en la Columna C. En nuestro ejemplo, $25-5=20$.

Si miramos en la TABLA DE PAGOS, cuando MI NIVEL DE EXTRACCIÓN es 5 (500) y el NIVEL DE EXTRACCIÓN DE ELLOS es 20 (2000), yo me gano 750 puntos. Ese número lo anoto en la Columna D (cuarta columna) de la 
HOJA DE DECISIONES. Ese número corresponde a lo que usted ganó en esa ronda, el cual deberá ser multiplicado por 0,9 (tasa de cambio) para convertir esos puntos en dinero real, por ejemplo si usted ganó 750 en una ronda deberá multiplicarlo por $0,9,750 \times 0,9=675$, es decir, en esta ronda usted ganó 675 pesos chilenos.

Es muy importante reiterar que nadie podrá, excepto el monitor, conocer el número que cada uno de ustedes decida en cada una de las rondas. El monitor solo anunciará el total del grupo, por lo cual usted no sabrá exactamente cómo jugó cada participante de su grupo.

Revisemos los pasos con un nuevo ejemplo.

Cada uno de ustedes decida ahora su nivel de extracción de locos para una ronda, anótelo en la Columna A de la HOJA DE DECISIONES y en una de las TARJETAS DE DECISIÓN, y levante la mano cuando termine para que los facilitadores retiren las tarjetas (puede ocupar ese momento para realizar alguna pregunta a los facilitadores).

En esta ronda de práctica el total del grupo fue ; en este momento, cada uno de ustedes debe anotar este número en la Columna $\mathrm{B}$, y proceder a restarle su decisión individual (Columna A) y anotar el resultado en la Columna C. Con estos datos (Columna A y Columna C) puede entonces obtener sus ganancias individuales en la TABLA DE PAGOS.

Esperamos que estos ejemplos le ayuden a entender cómo funciona la actividad de toma de decisiones, y cómo puede tomarlas respecto de su NIVEL DE EXTRACCIÓN DE LOCOS en cada ronda. Si en este momento tiene alguna pregunta acerca de cómo se ganan puntos con la toma de decisiones, por favor levante la mano y háganos saberlo.

[... MONITOR: pausa para resolver preguntas...]

\section{Preparación para comenzar con la toma de decisiones.}

Tres ejemplos de cómo funciona una TABLA DE PAGOS. Suponga que los puntos que obtiene en cada decisión están dados por la tabla siguiente (abajo). Recuerde que en cada ronda usted gana puntos según como extraiga el recurso loco.

\section{Ejemplo 1:}

Suponga que decide extraer 5 (que corresponde a 500) de sus 8 (que corresponden a 800) unidades del recurso loco. Suponga además que el monitor luego de sumar las unidades de todo el grupo, indicó que en total extrajeron 26 (2600) unidades de loco, por lo cual los demás miembros de su grupo, en conjunto, extrajeron 21 (que corresponden a 2100) unidades de loco, recuerde que al total de lo extraído por los cinco (5) integrantes del grupo debe restarle su propia extracción (en este caso $26-5=21$ ). Busque en la TABLA DE PAGOS la columna donde MI NIVEL DE EXTRACCIÓN DE LOCOS es 5, y la fila donde NIVEL DE EXTRACCIÓN DE es 21. En esta tabla y en la que utilizará, puede observar que usted ganaría 730 puntos por esta decisión. Recuerde que estos puntos deben ser multiplicados por 0,9 (tasa de cambio) para convertir esos puntos en dinero real, por lo cual en esta ronda al realizar la multiplicación usted ganaría 730 x 0,9 =657, es decir, en esta ronda usted ganaría 657 pesos chilenos. 
(Alta)

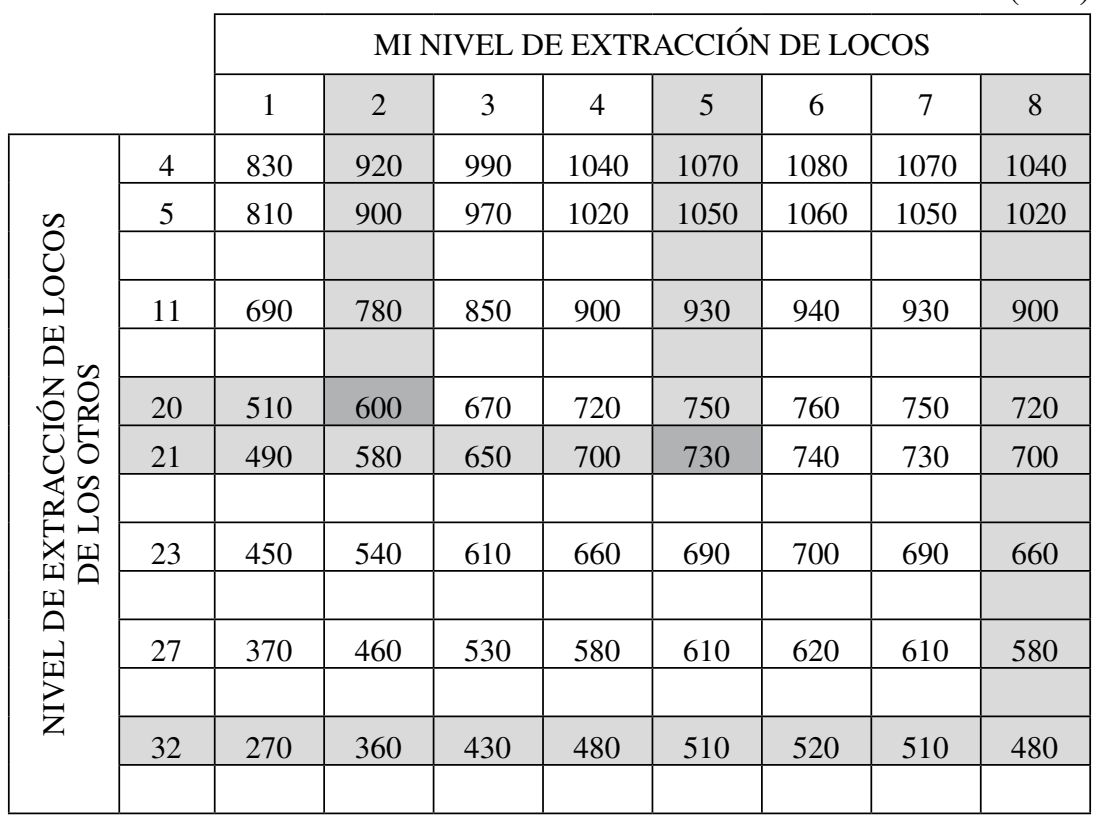

\section{Ejemplo 2:}

Suponga que usted decide reducir la extracción de locos a 2 (que corresponde a 200), es decir, en MI NIVEL DE EXTRACCIÓN DE LOCOS lo redujo a 2 (200) unidades. Suponga además que el monitor luego de sumar las unidades de todo el grupo, indicó que en total extrajeron 25 (2500) unidades de loco, por lo cual los demás miembros de su grupo, en conjunto, sumaron 20 (200) en NIVEL DE EXTRACCIÓN DE LOCOS DE LOS OTROS (25-5=20). Por tanto, si busca en la tabla de pagos usted ganaría 600 puntos por esta decisión. Recuerde que estos puntos deben ser multiplicados por 0,9 (tasa de cambio) por lo que usted ganaría $600 \times 0,9=540$ pesos chilenos.

Ejemplo 3:

Suponga, en cambio, que usted decide que MI NIVEL DE EXTRACCIÓN DE LOCOS sean 8 (800) unidades, y que además el monitor luego de sumar las unidades de todo el grupo, indicó que en total extrajeron 40 (4000) unidades de loco, por lo cual los demás miembros de su grupo, en conjunto extrajeron 32 (3200), es decir, el NIVEL DE EXTRACCIÓN DE LOCOS DE LOS OTROS es 32 (3200) (40-8=32). Los puntos que usted ganaría en esa ronda serían, esta vez, de 480 puntos por su decisión. Recuerde que estos puntos deben ser multiplicados por 0,9 (tasa de cambio) por lo que usted ganaría 480 × 0,9=432 pesos chilenos.

Para comenzar a tomar decisiones, organizaremos los asientos en una rueda donde todos miran hacia adentro. A partir de este momento se permitirá la comunicación entre los miembros del grupo, permitiendo la discusión del grupo durante un máximo de 5 minutos antes de tomar las decisiones de extracción, durante los cuales no podrán realizar acuerdos de transferencias de puntos o 
ganancias después de terminada la sesión. Una vez terminados los 5 minutos, organizaremos los asientos en una rueda donde todos miran hacia afuera, no volviendo a comunicarse entre ustedes en las siguientes rondas.

Si tienen preguntas, por favor háganlas en este momento. Solo cuando todos hayan entendido completamente, podremos iniciar.

\section{¿Hay preguntas?}

Si no existen más preguntas, les vamos a pedir que mantengamos silencio de ahora en adelante.

\section{Instrucciones tratamientos}

(El monitor y facilitadores deberán entregar las nuevas instrucciones y materiales (ver anexo 1, materiales experimentos letra f), además de colocar un resumen de las instrucciones en un lugar visible. Leer tratamiento B y luego chequear las dudas ¿Hay preguntas?)

\section{f. Tratamiento B: Fiscalización formal}

Además de las reglas descritas en las instrucciones que explicamos al inicio de la sesión, hay una regla adicional que explicaremos a continuación:

En las rondas anteriores la extracción dependía de sus decisiones respecto de la extracción del recurso loco, de 1 centena (100 unidades) esfuerzo mínimo a 8 centenas (800 unidades) como esfuerzo máximo que cada uno de ustedes podía realizar. Ahora les pediremos que piensen en la siguiente situación:

Usted forma parte de una organización constituida por 5 integrantes de la comunidad y realizaron la solicitud de un área de manejo localizada en el mismo lugar donde tradicionalmente han extraído locos, otorgándoles el área en el sector que ustedes solicitaron. Además les autorizan una cuota global a extraer por la organización en cada temporada, que en este caso supondremos será la misma en las próximas rondas correspondiente a 10 centenas (equivalentes a 1000 unidades de loco).

Para cumplir con la cuota otorgada, la autoridad decide dividir la cuota de manera individual en cuotas no transferibles, por lo que ustedes deberían extraer hasta 2 centenas ( 200 unidades) de loco por ronda para cumplir con la regulación. Sin embargo, usted tiene la misma capacidad de extracción que antes, es decir, de 1 centena ( 100 unidades) a 8 centenas ( 800 unidades), por lo cual usted debe decidir si cumple o no con la cuota otorgada.

Adicionalmente la autoridad ha instaurado un sistema de fiscalización en la zona con el fin de monitorear su extracción individual, de esta forma, en el caso de que usted sea fiscalizado y en la inspección sea sorprendido con más de 2 centenas (200 unidades) de loco, se le aplicará una multa en puntos por cada unidad adicional que sobrepase la cuota individual.

La tarea de fiscalización estará a cargo de un inspector el cual podrá inspeccionar solo 1 pescador por ronda, para lo cual seleccionará de manera aleatoria (al azar) a un integrante de la organización y procederá a inspeccionar su extracción. El procedimiento de selección del pescador a inspeccionar consiste en sacar desde una urna una de las 5 fichas que contienen los números de identificación entregados inicialmente (confidenciales). Si el inspector procede a inspeccionarlo y usted ha extraído más de 2 centenas ( 200 unidades) de loco, 
usted recibirá una multa que asciende a 200 puntos por cada centena adicional que extraiga del área de manejo. Si el inspector procede a la inspección y usted cumple con la regulación, usted no pagará multa.

En el momento que el número de la persona fiscalizada sea anunciado, el miembro del grupo que tiene dicho número no debe identificarse, para asegurar que el número asignado a cada miembro del grupo continuará siendo anónimo. Finalmente cabe consignar que la decisión del miembro de la organización será anunciada públicamente.

En resumen, de la misma manera que en rondas anteriores, usted podrá escoger su nivel de extracción entre 1 centena (100) y 8 centenas (800). Una vez que los cinco miembros de la organización hayan realizado su decisión, y que cada uno ha calculado sus ganancias, se procederá a seleccionar a un solo miembro de la organización para hacer la inspección y aplicar multa si corresponde.

Por ejemplo: Si usted eligió extraer 6 centenas de loco (600 unidades), es decir, extrajo 4 centenas (400 unidades) sobre la cuota fijada por la autoridad (2 centenas), con lo cual usted había ganado 1000 puntos, y usted no es seleccionado para la inspección, usted habría ganado los 1000 puntos, es decir, 1000x0, 9=900 pesos chilenos, sin embargo si es seleccionado para ser fiscalizado, el monitor procederá a aplicarle la multa correspondiente a 4 X200 $=800$, es decir, le restará 800 puntos del total de puntos que había ganado en esa ronda, es decir, $1000-800=200$ puntos, por lo cual usted ganaría 180 pesos chilenos.

Usted deberá analizar si cumple o no con la regulación. Recuerde que solo un miembro de la organización será inspeccionado en cada ronda por lo cual usted podría ser inspeccionado una o más veces, o bien en ninguna ronda.

\section{TABLA DE MULTAS}

\begin{tabular}{|llllllllll|}
\hline $\begin{array}{l}\text { Si sale escogido, } \\
\text { y su NIVEL DE } \\
\text { EXTRACCIÓN es de: }\end{array}$ & $1(100)$ & $2(200)$ & $3(300)$ & $4(400)$ & $5(500)$ & $6(600)$ & $7(700)$ & $8(800)$ \\
\hline $\begin{array}{l}\text { La multa que debe } \\
\text { pagar es de: }\end{array}$ & $-\$ 0$ & $-\$ 0$ & $-\$ 200$ & $-\$ 400$ & $-\$ 600$ & $-\$ 800$ & $-\$ 1000$ & $-\$ 1200$ \\
\hline
\end{tabular}

Para comenzar a tomar decisiones en esta nueva ronda, organizaremos los asientos en una rueda donde todos miran hacia adentro. A partir de este momento se permitirá la comunicación entre los miembros del grupo, permitiendo la discusión del grupo durante un máximo de 5 minutos antes de tomar las decisiones de extracción, durante los cuales no podrán realizar acuerdos de transferencias de puntos o ganancias después de terminada la sesión. Una vez terminados los 5 minutos, organizaremos los asientos en una rueda donde todos miran hacia afuera, no volviendo a comunicarse entre ustedes en las siguientes rondas.

¿Hay alguna pregunta?

Adicionalmente se incorpora la ficha que debieron llenar los monitores con los resultados de los experimentos (ver anexo 1, materiales experimentos letra g). 


\section{Materiales Experimentos}

\section{a. Consentimiento informado (Experimento y encuesta)}

Usted ha sido invitado a participar en un estudio de investigación sobre el manejo de recursos naturales, específicamente en Áreas de Manejo y Explotación de Recursos Bentónicos (AMERB) de la Región del Biobío, Chile. En este estudio es muy importante la participación de personas como usted, que por su trabajo diario está en contacto con el manejo de recursos naturales. Gracias a su participación en el siguiente juego, y posteriormente en una encuesta, podremos recoger información relevante sobre los pescadores artesanales y el sistema de normas existente en las áreas de manejo.

Usted podrá recibir premios de acuerdo con los puntajes que gane. Al final del juego deberá contestar algunas preguntas acerca del juego. Tanto la entrega de premios como la información sobre la forma como jugó cada participante en el juego se mantendrá en privado y para fines puramente académicos.

La duración aproximada de la sesión de juego de hoy es de una hora y media. Posteriormente, después de finalizado el juego, realizaremos una encuesta cuya duración aproximada será de cuarenta y cinco (45) minutos. Una vez finalizada la encuesta discutiremos algunos de los resultados del juego, además de entregar los premios que cada participante ganó durante el juego.

Su participación en este estudio es totalmente voluntaria. Usted puede retirarse en cualquier momento. Sin embargo, si decide retirarse, no podrá recibir premios, pues se necesita jugar las rondas completas del juego para acumular los puntos suficientes y contestar íntegramente la encuesta.

Esta investigación preservará la confidencialidad de su identidad, eliminando de las bases de datos toda la información sensible que pudiera servir para su identificación. Los datos se usarán solo con propósitos académicos codificando la información y manteniéndola en archivos seguros y resguardados en bases de datos a la que tendrán acceso solo los investigadores responsables de la presente investigación.

Los resultados del estudio serán usados para fines académicos y no se revelarán datos sensibles de los sujetos participantes. El financiamiento del estudio proviene del Programa Latinoamericano y del Caribe de Capacitación en Economía Ambiental-LACEEP.

ACEPTACIÓN (escriba con letra imprenta)

Yo, declaro que:

Comprendo la información anterior, así como mis derechos y compromisos durante el estudio.

He tenido la oportunidad de hacer preguntas acerca del propósito y procedimientos en relación con el estudio.

Mi participación en esta investigación es voluntaria. Reconozco que puedo negarme a participar, o renunciar a participar en cualquier momento y renunciar a recibir premios al final.

Si en algún momento tengo alguna pregunta relacionada con la investigación o mi participación, puedo contactarme con el investigador, Oscar Santis Brante, quien responderá mis preguntas. El teléfono del investigador es (56-9) XXXXXX.Y su correo electrónico es osantis@udec.cl 
Certifico también que he recibido una copia del presente consentimiento informado.

Mi firma significa que estoy de acuerdo con participar en este estudio.

Firma del Participante:

Fecha:

Nombre

b. Tarjeta con número de identificación

\begin{tabular}{|ll|}
\hline & Experimento: \\
& \\
$N^{\text {o }}$ Identificación: $A$ & Timbre \\
& \\
& \\
\hline
\end{tabular}

c. Tablas de pagos

TABLA DE PAGOS (ABUNDANCIA)

\begin{tabular}{|c|c|c|c|c|c|c|c|c|c|}
\hline \multicolumn{10}{|c|}{ MI NIVEL DE EXTRACCIÓN DE LOCOS } \\
\hline \multirow{30}{*}{ 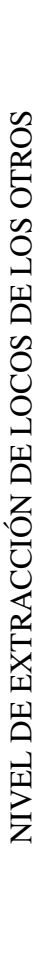 } & Total & $\mathbf{1}(100)$ & $2(200)$ & 3(300) & $4(400)$ & $5(500)$ & $6(600)$ & $7(700)$ & $8(800)$ \\
\hline & $4(400)$ & 830 & 920 & 990 & 1040 & 1070 & 1080 & 1070 & 1040 \\
\hline & $\mathbf{5}(500)$ & 810 & 900 & 970 & 1020 & 1050 & 1060 & 1050 & 1020 \\
\hline & $6(600)$ & 790 & 880 & 950 & 1000 & 1030 & 1040 & 1030 & 1000 \\
\hline & $7(700)$ & 770 & 860 & 930 & 980 & 1010 & 1020 & 1010 & 980 \\
\hline & $8(800)$ & 750 & 840 & 910 & 960 & 990 & 1000 & 990 & 960 \\
\hline & $9(900)$ & 730 & 820 & 890 & 940 & 970 & 980 & 970 & 940 \\
\hline & $\mathbf{1 0}(1000)$ & 710 & 800 & 870 & 920 & 950 & 960 & 950 & 920 \\
\hline & 11(1100) & 690 & 780 & 850 & 900 & 930 & 940 & 930 & 900 \\
\hline & 12(1200) & 670 & 760 & 830 & 880 & 910 & 920 & 910 & 880 \\
\hline & 13(1300) & 650 & 740 & 810 & 860 & 890 & 900 & 890 & 860 \\
\hline & 14(1400) & 630 & 720 & 790 & 840 & 870 & 880 & 870 & 840 \\
\hline & $\mathbf{1 5}(1500)$ & 610 & 700 & 770 & 820 & 850 & 860 & 850 & 820 \\
\hline & 16(1600) & 590 & 680 & 750 & 800 & 830 & 840 & 830 & 800 \\
\hline & 17(1700) & 570 & 660 & 730 & 780 & 810 & 820 & 810 & 780 \\
\hline & 18(1800) & 550 & 640 & 710 & 760 & 790 & 800 & 790 & 760 \\
\hline & 19(1900) & 530 & 620 & 690 & 740 & 770 & 780 & 770 & 740 \\
\hline & 20(2000) & 510 & 600 & 670 & 720 & 750 & 760 & 750 & 720 \\
\hline & $\mathbf{2 1}(2100)$ & 490 & 580 & 650 & 700 & 730 & 740 & 730 & 700 \\
\hline & $\mathbf{2 2}(2200)$ & 470 & 560 & 630 & 680 & 710 & 720 & 710 & 680 \\
\hline & $\mathbf{2 3}(2300)$ & 450 & 540 & 610 & 660 & 690 & 700 & 690 & 660 \\
\hline & $\mathbf{2 4}(2400)$ & 430 & 520 & 590 & 640 & 670 & 680 & 670 & 640 \\
\hline & $\mathbf{2 5}(2500)$ & 410 & 500 & 570 & 620 & 650 & 660 & 650 & 620 \\
\hline & $\mathbf{2 6}(2600)$ & 390 & 480 & 550 & 600 & 630 & 640 & 630 & 600 \\
\hline & $\mathbf{2 7}(2700)$ & 370 & 460 & 530 & 580 & 610 & 620 & 610 & 580 \\
\hline & $\mathbf{2 8}(2800)$ & 350 & 440 & 510 & 560 & 590 & 600 & 590 & 560 \\
\hline & 29(2900) & 330 & 420 & 490 & 540 & 570 & 580 & 570 & 540 \\
\hline & 30(3000) & 310 & 400 & 470 & 520 & 550 & 560 & 550 & 520 \\
\hline & 31(3100) & 290 & 380 & 450 & 500 & 530 & 540 & 530 & 500 \\
\hline & 32(3200) & 270 & 360 & 430 & 480 & 510 & 520 & 510 & 480 \\
\hline
\end{tabular}


TABLA DE PAGOS (ESCASEZ)

\begin{tabular}{|c|c|c|c|c|c|c|c|c|c|}
\hline \multicolumn{10}{|c|}{ MI NIVEL DE EXTRACCIÓN DE LOCOS } \\
\hline & Total & $\mathbf{1}(100)$ & $2(200)$ & $3(300)$ & $4(400)$ & $\mathbf{5}(500)$ & $6(600)$ & 7(700) & $8(800)$ \\
\hline \multirow{29}{*}{ 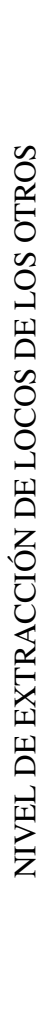 } & $4(400)$ & 820 & 880 & 900 & 880 & 820 & 720 & 580 & 400 \\
\hline & $\mathbf{5}(500)$ & 800 & 860 & 880 & 860 & 800 & 700 & 560 & 380 \\
\hline & 6(600) & 780 & 840 & 860 & 840 & 780 & 680 & 540 & 360 \\
\hline & 7(700) & 760 & 820 & 840 & 820 & 760 & 660 & 520 & 340 \\
\hline & $8(800)$ & 740 & 800 & 820 & 800 & 740 & 640 & 500 & 320 \\
\hline & $9(900)$ & 720 & 780 & 800 & 780 & 720 & 620 & 480 & 300 \\
\hline & $\mathbf{1 0}(1000)$ & 700 & 760 & 780 & 760 & 700 & 600 & 460 & 280 \\
\hline & $\mathbf{1 1}(1100)$ & 680 & 740 & 760 & 740 & 680 & 580 & 440 & 260 \\
\hline & $\mathbf{1 2}(1200)$ & 660 & 720 & 740 & 720 & 660 & 560 & 420 & 240 \\
\hline & $\mathbf{1 3}(1300)$ & 640 & 700 & 720 & 700 & 640 & 540 & 400 & 220 \\
\hline & 14(1400) & 620 & 680 & 700 & 680 & 620 & 520 & 380 & 200 \\
\hline & $\mathbf{1 5}(1500)$ & 600 & 660 & 680 & 660 & 600 & 500 & 360 & 180 \\
\hline & 16(1600) & 580 & 640 & 660 & 640 & 580 & 480 & 340 & 160 \\
\hline & 17(1700) & 560 & 620 & 640 & 620 & 560 & 460 & 320 & 140 \\
\hline & 18(1800) & 540 & 600 & 620 & 600 & 540 & 440 & 300 & 120 \\
\hline & 19(1900) & 520 & 580 & 600 & 580 & 520 & 420 & 280 & 100 \\
\hline & $\mathbf{2 0}(2000)$ & 500 & 560 & 580 & 560 & 500 & 400 & 260 & 80 \\
\hline & $\mathbf{2 1}(2100)$ & 480 & 540 & 560 & 540 & 480 & 380 & 240 & 60 \\
\hline & $22(2200)$ & 460 & 520 & 540 & 520 & 460 & 360 & 220 & 40 \\
\hline & $\mathbf{2 3}(2300)$ & 440 & 500 & 520 & 500 & 440 & 340 & 200 & 20 \\
\hline & $\mathbf{2 4}(2400)$ & 420 & 480 & 500 & 480 & 420 & 320 & 180 & 0 \\
\hline & $\mathbf{2 5}(2500)$ & 400 & 460 & 480 & 460 & 400 & 300 & 160 & -20 \\
\hline & $\mathbf{2 6}(2600)$ & 380 & 440 & 460 & 440 & 380 & 280 & 140 & -40 \\
\hline & $\mathbf{2 7}(2700)$ & 360 & 420 & 440 & 420 & 360 & 260 & 120 & -60 \\
\hline & $\mathbf{2 8}(2800)$ & 340 & 400 & 420 & 400 & 340 & 240 & 100 & -80 \\
\hline & $\mathbf{2 9}(2900)$ & 320 & 380 & 400 & 380 & 320 & 220 & 80 & -100 \\
\hline & $\mathbf{3 0}(3000)$ & 300 & 360 & 380 & 360 & 300 & 200 & 60 & -120 \\
\hline & 31(3100) & 280 & 340 & 360 & 340 & 280 & 180 & 40 & -140 \\
\hline & $32(3200)$ & 260 & 320 & 340 & 320 & 260 & 160 & 20 & -160 \\
\hline
\end{tabular}

\section{d. Tarjetas de decisión}

TARJETA DE DECISIÓN

\begin{tabular}{|l|c|}
\hline Número del pescador & A \\
\hline Número de ronda & $\ldots \ldots$ \\
\hline $\begin{array}{l}\text { Mi nivel de extracción } \\
\text { Elegir entre: } \\
1 \text { centena (100 unidades) } \\
\text { a } 8 \text { centenas (800 unidades) }\end{array}$ & $\ldots \ldots$ \\
\hline
\end{tabular}




\section{e. Hoja de decisiones}

PESCADOR NÚMERO:

Fecha: / I

TABLA DE PAGOS: Alta__ Baja

Hora: a.m. / p.m. Lugar:

\begin{tabular}{|l|l|l|l|l|}
\hline & A & B & C & D \\
\hline Ronda & $\begin{array}{c}\text { Mi nivel de extrac- } \\
\text { ción de Locos (mi } \\
\text { decisión) }\end{array}$ & $\begin{array}{c}\text { Total extraído por } \\
\text { el grupo (lo anun- } \\
\text { cia el monitor) }\end{array}$ & $\begin{array}{c}\text { El nivel de extrac- } \\
\text { ción de Locos de } \\
\text { los otros (columna } \\
\text { B menos columna } \\
\text { A) }\end{array}$ & $\begin{array}{c}\text { Mis ganancias en } \\
\text { esta ronda (use la } \\
\text { Tabla de Pagos) }\end{array}$ \\
\hline Práctica & & & & \\
\hline Práctica & & & & \\
\hline Práctica & & & & \\
\hline 1 & & & & \\
\hline 2 & & & & \\
\hline 3 & & & & \\
\hline 4 & & & & \\
\hline 5 & & & & \\
\hline 6 & & & & \\
\hline 7 & & & & \\
\hline 8 & & & & \\
\hline 9 & & & & \\
\hline 10 & & & & \\
\hline & & & & \\
\hline & & & & \\
\hline & & & & \\
\hline & & & & \\
\hline & & & & \\
\hline & & & & \\
\hline & & & & \\
\hline & & & & \\
\hline & & & & \\
\hline & & & & \\
\hline
\end{tabular}




\section{f. Hoja de decisiones (Segunda etapa Formal)}

PESCADOR NÚMERO: TABLA DE PAGOS:

Fecha: / / Hora: a.m. / p.m. Lugar:

\begin{tabular}{|c|c|c|c|c|c|c|}
\hline & A & B & $\mathrm{C}$ & D & E & $\mathrm{F}$ \\
\hline Ronda & $\begin{array}{c}\text { Mi nivel de } \\
\text { Extracción } \\
\text { de Locos } \\
\text { (mi } \\
\text { decisión) }\end{array}$ & $\begin{array}{c}\text { Total extraí- } \\
\text { do por el } \\
\text { grupo }\end{array}$ & $\begin{array}{c}\text { El nivel de } \\
\text { Extracción } \\
\text { de Locos } \\
\text { de los otros } \\
\text { (columna } \\
\text { B menos co- } \\
\text { lumna A) }\end{array}$ & $\begin{array}{l}\text { Mis ga- } \\
\text { nancias } \\
\text { antes de la } \\
\text { regulación }\end{array}$ & $\begin{array}{l}\text { Mi multa en } \\
\text { esta ronda }\end{array}$ & $\begin{array}{l}\text { Mis ganan- } \\
\text { cias finales } \\
\text { en esta } \\
\text { ronda } \\
\text { (columna D } \\
\text { menos } \\
\text { columna E) }\end{array}$ \\
\hline \multicolumn{7}{|l|}{1} \\
\hline \multicolumn{7}{|c|}{2} \\
\hline \multicolumn{7}{|l|}{3} \\
\hline \multicolumn{7}{|l|}{4} \\
\hline \multicolumn{7}{|l|}{5} \\
\hline \multicolumn{7}{|l|}{6} \\
\hline \multicolumn{7}{|l|}{7} \\
\hline \multicolumn{7}{|l|}{8} \\
\hline \multicolumn{7}{|l|}{9} \\
\hline \multicolumn{7}{|l|}{10} \\
\hline \multicolumn{7}{|l|}{11} \\
\hline \multicolumn{7}{|l|}{12} \\
\hline & & & & & & \\
\hline & & & & & & \\
\hline & & & & & & \\
\hline Total & & & & & & \\
\hline
\end{tabular}

TABLA DE MULTAS

\begin{tabular}{|l|c|c|c|c|c|c|c|c|}
\hline $\begin{array}{l}\text { Si sale escogido, } \\
\text { y su NIVEL DE } \\
\text { EXTRACCIÓN es de: }\end{array}$ & $1(100)$ & $2(200)$ & $3(300)$ & $4(400)$ & $5(500)$ & $6(600)$ & $7(700)$ & $8(800)$ \\
\hline $\begin{array}{l}\text { La multa que debe } \\
\text { pagar es de: }\end{array}$ & $-\$ 0$ & $-\$ 0$ & $-\$ 200$ & $-\$ 400$ & $-\$ 600$ & $-\$ 800$ & $-\$ 1000$ & $-\$ 1200$ \\
\hline
\end{tabular}




\section{g. Ficha para el monitor}

Fecha: __ I_ Hora: a.m. / p.m. Lugar:

\begin{tabular}{|c|c|c|c|c|c|c|c|c|c|}
\hline & & & $\mathrm{FU}$ & $\begin{array}{l}\text { DEC } \\
\text { ERZ }\end{array}$ & $\begin{array}{l}\text { ISIC } \\
\text { O D }\end{array}$ & $\begin{array}{l}\mathrm{ON} \\
\mathrm{E} \mathrm{E}\end{array}$ & $\begin{array}{l}\text { ES INDIV } \\
\text { XTRACC }\end{array}$ & $\begin{array}{l}\text { IDUALES } \\
\text { IÓN DE LOCOS }\end{array}$ & \\
\hline & & & & cado & & & TOTAL & & \\
\hline Ronda & Regla & 1 & 2 & 3 & 4 & 5 & GRUPO & & \\
\hline Práctica & & & & & & & & & \\
\hline Práctica & & & & & & & & & \\
\hline Práctica & & & & & & & & & \\
\hline 1 & $X$ & & & & & & & & \\
\hline 2 & $X$ & & & & & & & & \\
\hline 3 & $x$ & & & & & & & & \\
\hline 4 & $X$ & & & & & & & & \\
\hline 5 & $x$ & & & & & & & & \\
\hline 6 & $X$ & & & & & & & & \\
\hline 7 & $X$ & & & & & & & & \\
\hline 8 & $X$ & & & & & & & & \\
\hline 9 & $X$ & & & & & & & & \\
\hline 10 & $\mathrm{X}$ & & & & & & & & \\
\hline Etapa 2 & Regla & & $\begin{array}{l}\mathrm{SCl} \\
\mathrm{CCI} \\
\mathrm{ESS}\end{array}$ & $\begin{array}{l}\text { IÓI } \\
\text { CAI }\end{array}$ & $\begin{array}{l}\mathrm{LA} \\
\mathrm{DE} \\
\mathrm{OR}\end{array}$ & & $\begin{array}{l}\text { TOTAL } \\
\text { GRUPO }\end{array}$ & $\begin{array}{l}\text { número de } \\
\text { Pescador } \\
\text { inspeccionado }\end{array}$ & Sanción \\
\hline & & 1 & 2 & 3 & 4 & 5 & & & \\
\hline 11 & & & & & & & & & \\
\hline 12 & & & & & & & & & \\
\hline 13 & & & & & & & & & \\
\hline 14 & & & & & & & & & \\
\hline 15 & & & & & & & & & \\
\hline 16 & & & & & & & & & \\
\hline 17 & & & & & & & & & \\
\hline 18 & & & & & & & & & \\
\hline 19 & & & & & & & & & \\
\hline 20 & & & & & & & & & \\
\hline Total & & & & & & & & & \\
\hline
\end{tabular}

